\title{
Orbital and magnetic ordering and domain-wall conduction in ferrimagnet $\mathrm{La}_{5} \mathrm{Mo}_{4} \mathrm{O}_{16}$
}

\author{
Takuro Katsufuji $\odot,{ }^{1,2}$ Masayuki Miyake, ${ }^{1}$ Makoto Naka, ${ }^{3}$ Masahito Mochizuki $\odot,{ }^{1}$ Sota Kogo, ${ }^{1}$ Tomomasa Kajita $\odot$, \\ Yasuhiro Shimizu $\odot,{ }^{4}$ Masayuki Itoh, ${ }^{4}$ Takatoshi Hasegawa, ${ }^{1}$ Shunsuke Shimose, ${ }^{1}$ Shunta Noguchi, ${ }^{1}$ Takuo Saiki, ${ }^{1}$ \\ Takuro Sato, ${ }^{5}$ and Fumitaka Kagawa ${ }^{6,5}$ \\ ${ }^{1}$ Department of Physics, Waseda University, Tokyo 169-8555, Japan \\ ${ }^{2}$ Kagami Memorial Research Institute for Materials Science and Technology, Waseda University, Tokyo 169-0051, Japan \\ ${ }^{3}$ Waseda Institute for Advanced Study, Waseda University, Tokyo, 169-8050, Japan \\ ${ }^{4}$ Department of Physics, Nagoya University, Chikusa, Nagoya 464-8602, Japan \\ ${ }^{5}$ RIKEN Center for Emergent Matter Science (CEMS), Wako 351-0198, Japan \\ ${ }^{6}$ Department of Applied Physics, University of Tokyo, Tokyo 113-8656, Japan
}

(Received 14 July 2020; revised 11 November 2020; accepted 6 January 2021; published 2 February 2021)

\begin{abstract}
We studied the ferrimagnet $\mathrm{La}_{5} \mathrm{Mo}_{4} \mathrm{O}_{16}$ with $\mathrm{Mo}$ ions on quasisquare lattices both experimentally and theoretically. We found that in addition to the antiferromagnetic ordering at $T_{N}=200 \mathrm{~K}$, a magnetic phase transition into a ferrimagnetic phase occurs at $T_{s 1}=60 \mathrm{~K}$, which is caused by the orbital ordering of the Mo $4 d$ states. We also found that the relatively small size of ferrimagnetic domains despite the large Ising anisotropy of the Mo spins are formed below $T_{s 1}$, which are responsible for the relaxation behavior and the memory effect in the magnetization. Nonvolatile positive magnetoresistance observed in this compound below $T_{s 1}$ can be attributed to the fact that such magnetic domains and the electrical conduction along the domain walls disappear with the applied magnetic field.
\end{abstract}

DOI: 10.1103/PhysRevResearch.3.013105

\section{INTRODUCTION}

Domain walls are the objects existing between two regions (i.e., domains) with different directions of the order parameter in the ordered state. The control of domain structures is important in various ordered materials, such as ferromagnets, permanent magnets, and ferroelectric materials [1-3]. In such materials, however, the domain walls act only as the objects accompanied by the domains. In some cases, the domain wall itself has an important physical property, for example, the electrical conduction along the domain walls [4-12]. Namely, even though the material in the ordered state is insulating as a bulk property, electrical conduction is possible in some cases along the boundaries of the domains, i.e., domain walls. Such a phenomenon was first discovered in the domain walls of ferroelectrics, $\mathrm{BiFeO}_{3}[4,5]$ and hexagonal $R \mathrm{MnO}_{3}$ [6,7], and then in those of antiferromagnets, $R_{2} \operatorname{Ir}_{2} \mathrm{O}_{7}[8,9]$ and $\mathrm{Cd}_{2} \mathrm{Os}_{2} \mathrm{O}_{7}$ [11] with a pyrochlore structure. In particular, the topological nature of domain-wall conduction was proposed for $R_{2} \operatorname{Ir}_{2} \mathrm{O}_{7}$ [10], which is a candidate Weyl semimetal. As a similar phenomenon, chiral edge conduction on domain walls in the quantum anomalous Hall state of a magnetic topological insulator was demonstrated experimentally [12].

Published by the American Physical Society under the terms of the Creative Commons Attribution 4.0 International license. Further distribution of this work must maintain attribution to the author(s) and the published article's title, journal citation, and DOI.
One of the difficulties in detecting the electrical conduction along the domain walls of antiferromagnets is to distinguish it from a bulk conduction since it is not easy to control the domain structures of antiferromagnets with a low magnetic field. Indeed, the magnetic domains in pyrochlore magnets discussed above was controlled in such a way that the temperature of the sample is reduced with a fixed applied magnetic field ("trained") or a relatively large magnetic field is applied with a fixed temperature so that the antiferromagnetic domain structures are partially suppressed and the domainwall conduction is also suppressed. Although the positive magnetoresistance by such a procedure has been observed experimentally, it is unclear whether it is really caused by domain-wall conduction or magnetoresistance as bulk properties. To clarify this issue, microwave impedance microscopy was employed for $R_{2} \mathrm{Ir}_{2} \mathrm{O}_{7}$ [8], which can directly image the metallic conduction paths, and convincing evidence of the domain-wall conduction was obtained. Nevertheless, it is preferable to find the material with domain-wall conduction, the domain structures of which can be easily modified with a low magnetic field.

In this respect, it may sound a good idea to work on ferromagnets to study the electrical conduction along domain walls. However, the domain walls in ferromagnets have the antiferromagnetic spin configuration and thus, it is unlikely that the electrical conduction along the domain walls is higher than the bulk electrical conduction. This dilemma can be resolved by working on ferrimagnets, where the domain structures can be easily suppressed with a low magnetic field because of the existence of spontaneous magnetization, whereas the domain walls have a ferromagnetic spin 


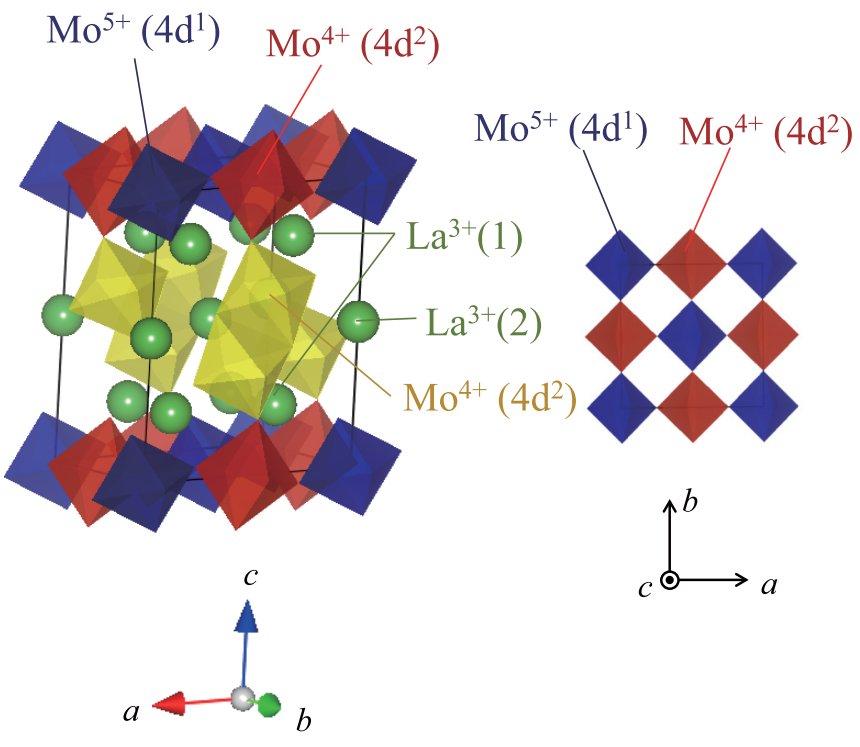

FIG. 1. Crystal structure of $\mathrm{La}_{5} \mathrm{Mo}_{4} \mathrm{O}_{16}$ viewed from the side (left) and top (right) drawn by VESTA [27].

configuration, which possibly yields higher electrical conduction than the bulk conduction dominated by the antiferromagnetic spin configuration. In this case, positive magnetoresistance with a low magnetic field caused by the disappearance of the conducting magnetic domain walls is expected.

In this study, we investigated the transport and magnetic properties of $\mathrm{La}_{5} \mathrm{Mo}_{4} \mathrm{O}_{16}$ [13-22] with a ferrimagnetic configuration of Mo spins as a candidate material exhibiting electrical conduction along magnetic domain walls.

$\mathrm{La}_{5} \mathrm{Mo}_{4} \mathrm{O}_{16}$ (space group $C 2 / m$ ) consists of quasisquare lattices of Mo ions along the $a b$ plane, where $\mathrm{Mo}^{4+}\left(4 d^{2}\right)$ and $\mathrm{Mo}^{5+}\left(4 d^{1}\right)$ ions are arranged in a checkerboard pattern (Fig. 1). $\mathrm{A} \mathrm{Mo}^{4+}$ in one layer of the square lattice and another $\mathrm{Mo}^{4+}$ in the next layer are connected by a $\mathrm{Mo}_{2} \mathrm{O}_{10}$ pillar with nonmagnetic $\mathrm{Mo}^{4+}$ pairs. It is known that several other compounds take a similar crystal structure [23-26]. The electrical resistivity $\rho$ of $\mathrm{La}_{5} \mathrm{Mo}_{4} \mathrm{O}_{16}$ increases with decreasing temperature $T$ and diverges at the lowest $T$, indicating an insulating character. This compound exhibits an antiferromagnetic ordering below $T_{\mathrm{N}}=200 \mathrm{~K}$, where the $\mathrm{Mo}^{4+}$ and $\mathrm{Mo}^{5+}$ spins in the direction of the $c$ axis (the easy axis) are ordered antiferromagnetically, yielding a ferrimagnetic moment along the $c$ axis at each layer of the square lattice. These ferrimagnetic moments are antiferromagnetically aligned between the layers and form the interlayer antiferromagnetic (IL-AF) state below $T_{\mathrm{N}}$. Such antiferromagnetically aligned ferrimagnetic moments are flipped with an applied magnetic field of only $\sim 0.5 \mathrm{~T}$ along the $c$ axis, resulting in ferromagnetic alignment, called the interlayer ferromagnetic (IL-F) state. This means that the interlayer antiferromagnetic interaction is three orders of magnitude smaller than the intralayer antiferromagnetic interaction, indicating a highly two-dimensional character of the magnetism in this compound. Associated with such a metamagnetic phase transition, a decrease in the resistivity of several percent (negative magnetoresistance) is observed.

In addition to $T_{\mathrm{N}}=200 \mathrm{~K}$, there are other anomalies (phase transitions) at $T_{s 2}=95 \mathrm{~K}$ and $T_{s 1}=60 \mathrm{~K}$. With decreasing
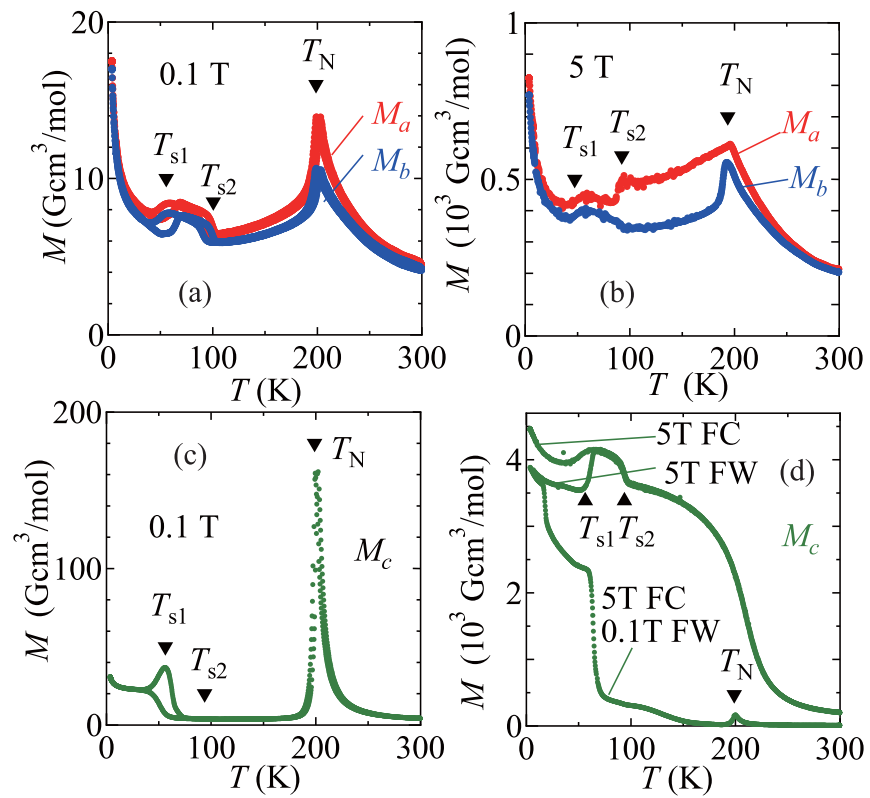

FIG. 2. Magnetization as a function of $T$ for $\mathrm{La}_{5} \mathrm{Mo}_{4} \mathrm{O}_{16}$ (a) along the $a$ and $b$ axes with $H=0.1 \mathrm{~T}$, (b) along the $a$ and $b$ axes with $H=5 \mathrm{~T}$, (c) along the $c$ axis with $H=0.1 \mathrm{~T}$, and (d) along the $c$ axis with $H=5 \mathrm{~T}$ in the field-cooling (FC) and field-warming (FW) runs, and with $H=0.1 \mathrm{~T}$ in the warming run after cooling the sample with applied $H=5 \mathrm{~T}$.

$T, \rho$ increases at $T_{s 2}$ but decreases at $T_{s 1}$. The magnetization $M$ also exhibits anomalies at $T_{s 2}$ and $T_{s 1}$. In particular, after decreasing $T$ with applied $H=5 \mathrm{~T}$ along the $c$ axis, $M$ measured at $H=0.1 \mathrm{~T}$ with increasing $T$ sharply decreases at $T_{s 1}$ [Fig. 2(d)], indicating the existence of a spontaneous magnetization below $T_{s 1}=60 \mathrm{~K}$. The $M(H)$ curve also changes from that without finite $M$ at $H=0$ above $T_{s 1}$ to that with a finite $M$ at $H=0$ after a finite $H$ is applied along the $c$ axis below $T_{s 1}$ (Fig. 3). However, it is unclear only from these measurements whether the initial magnetic state without $H$ is the ferromagnetic alignment of the ferrimagnetic moments (IL-F state) with the magnetic domains, or the antiferromagnetic alignment (IL-AF state), which changes to the IL-F state in a nonvolatile manner with applied $H$.

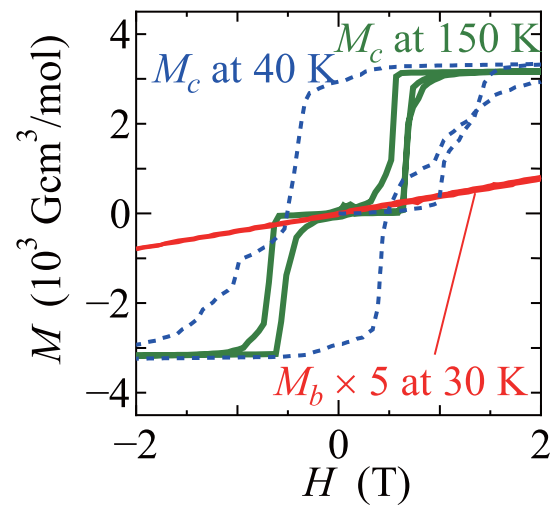

FIG. 3. Magnetization $M$ for $\mathrm{La}_{5} \mathrm{Mo}_{4} \mathrm{O}_{16}$ along the $c$ axis at $150 \mathrm{~K}$ (solid line) and at $40 \mathrm{~K}$ (dashed line) and along the $b$ axis at $30 \mathrm{~K}$ multiplied by five (solid line) as a function of $H$. 
One of the intriguing phenomena observed below $T_{s 1}=$ $60 \mathrm{~K}$ is the large nonvolatile positive magnetoresistance [21]. Namely, with increasing $H \| c, \rho$ increases almost twofold, and after that, even with a further sweep of $H$ between 5 and $-5 \mathrm{~T}, \rho$ barely changes. This behavior is quite different from the negative magnetoresistance for $T>T_{s 1}$, implying that the magnetic state below $T_{s 1}$ is no longer the IL-AF state. On the other hand, if the magnetic state below $T_{s 1}$ is the IL-F state, it is difficult to explain the magnetoresistance with the initial increase in $H$ as a bulk property since with applied $H$, only the direction of the spontaneous magnetization changes and there is no reason that the magnitude of the bulk resistivity would increase as a consequence. This implies that the existence of domain walls without magnetic field and their disappearance with applied magnetic field are responsible for the positive magnetoresistance. Another characteristic below $T_{s 1}$ is the relaxation (time-dependent) behavior of $\rho$ and $M$ when a magnetic field is applied [21]. This implies that the magnetic state below $T_{s 1}$ has a disordered character.

In this study, we performed detailed measurements of electrical resistivity, magnetization, and strain, and also performed nuclear magnetic resonance (NMR) measurements and magnetic-force microscopy (MFM) of $\mathrm{La}_{5} \mathrm{Mo}_{4} \mathrm{O}_{16}$. Compared with the previous studies [19-21], we focus on the anisotropy of not only those along the $a b$ plane and those perpendicular to the plane (along the $c$ axis), but also between the $a$ and $b$ axis within the plane to clarify the nature of each phase. We also performed the Hartree-Fock calculation for the present compound.

This paper is organized as follows: In Sec. II, the methods of the experiments are described. In Sec. III, the properties with specific anisotropy below $T_{N}$ (mostly above $T_{s 2}$ ) are discussed based on the results of the magnetization, resistivity, optical conductivity and magnetoresistance. In Sec. IV, the properties below $T_{s 2}$ and $T_{s 1}$ are discussed based on the results of NMR, magnetoresistance, and strain measurement. In Sec. V, the result of the magnetic-force microscope under the variation of $T$ and magnetic field $H$ and the memory effect in the magnetization are described focusing on its disorder character, and the relation between the magnetic domains and the magnetoresistance are discussed. In Sec. VI, the Hartree-Fock calculation on the present compound is described, and orbital ordering and the electrical conduction between the magnetic domains are investigated based on the calculation.

\section{EXPERIMENT}

The single crystals used in the study were grown by the floating-zone method. Details were described elsewhere [21]. We found that the anomalies at $T_{s 1}$ and $T_{s 2}$ are sensitive to the sample quality, and the crystals with higher $T_{N}(>200 \mathrm{~K})$ were chosen and used in the present study. The crystal axes ( $a$, $b$, and $c$ ) were determined by the Laue method. The crystals were typically rectangular with a size of $2 \mathrm{~mm} \times 2 \mathrm{~mm} \times$ $1 \mathrm{~mm}$. Magnetization was measured using a SQUID magnetometer. Both a two-point probes method and a four-point probes method were employed to measure the resistivity, by which resistivities of up to $\sim 10^{12}$ and up to $\sim 10^{8} \Omega \mathrm{cm}$ can be obtained, respectively. For both measurements, silver paste cured at room temperature was used as electrodes. The mag- netoresistance was measured with applied $H$ up to $5 \mathrm{~T}$ along the $c$ axis using a superconducting magnet. The strain of the sample was measured by a strain-gauge technique without and with an applied $H$ along the $c$ axis using the superconducting magnet. Optical reflectivity was measured between 0.08 and $0.8 \mathrm{eV}$ using an FTIR spectrometer and between 0.7 and $5 \mathrm{eV}$ using a grating spectrometer on the polished surface along the $a b$ plane.

NMR was conducted at a constant $H=9.089 \mathrm{~T}$. The frequency-swept ${ }^{139} \mathrm{La}$ NMR spectrum was obtained from spin echo measurements at $0.3 \mathrm{MHz}$ steps. The nuclear spinlattice relaxation rate $T_{1}^{-1}$ was measured by the saturation recovery method. The spin-echo decay rate $T_{2}^{-1}$ was also measured to detect the phase transition. The rf power for NMR experiments is about $10 \mathrm{~W}$, the pulse duration $\tau_{\pi / 2}=1 \mu \mathrm{s}$, and the time between pulses $\tau=10 \mu \mathrm{s}$. The coil was directly wound around the sample with dimensions $2 \mathrm{~mm} \times 2 \mathrm{~mm} \times$ $0.5 \mathrm{~mm}$.

MFM was performed on the polished $a b$ surface as used for the optical measurement by detecting the resonant frequency shift of the cantilever in the constant-height $(100 \mathrm{~nm})$ mode. The signal of the MFM in this compound was extremely small $(|\Delta f| \sim 1 \mathrm{~Hz}$ for $f=86 \mathrm{kHz})$ because of the small spontaneous magnetization caused by the ferrimagnetic layers of the $S=1$ and $S=1 / 2$ spins with a large distance between each other $(10.3 \AA)$, and thus, we took the image for the area of $\sim 1 \mu \mathrm{m} \times 1 \mu \mathrm{m}$ and subtracted the sum of a linear and a quadratic function of length as the back ground from the raw data, which is likely to arise from the surface roughness of the sample.

Note that for the measurements of $H$ dependence, $T$ of the sample is increased above $T_{\mathrm{N}}=200 \mathrm{~K}$ before every measurement so that it is performed in the initial magnetization process.

\section{PHYSICAL PROPERTIES BELOW $T_{\mathrm{N}}$}

Figure 2 shows the $T$ dependence of the magnetization measured at $H=0.1$ and $5 \mathrm{~T}$ along the $a, b$, and $c$ axes ( $M_{a}, M_{b}$, and $M_{c}$, respectively). With $H=0.1$ T [Figs. 2(a) and 2(c)], $M$ for all the directions behave similarly; a peak appears at $T_{\mathrm{N}}=200 \mathrm{~K}$, and small anomalies appear at $T_{s 2}=$ $95 \mathrm{~K}$ and $T_{s 1}=60 \mathrm{~K}$. On the other hand, as already reported in Ref. [21], $M_{c}$ at $5 \mathrm{~T}$ [Fig. 2(d)] behaves differently from that at $H=0.1 \mathrm{~T}$. Below $T_{\mathrm{N}}=200 \mathrm{~K}, M_{c}$ keeps increasing with decreasing $T$, and approaches $\sim 0.8 \mu_{B}$ per unit cell $\left(=5585 \times 0.8 \mathrm{Gcm}^{3} / \mathrm{mol}\right)$, indicating the ferromagnetic alignment of the ferrimagnetic moment between the layers. Indeed, as shown in Fig. 3, a metamagnetic phase transition occurs when $H$ is applied along the $c$ axis with a fixed $T$. On the other hand, $M_{a}$ and $M_{b}$ at $H=5 \mathrm{~T}$ [Fig. 2(b)] behave similarly to those at $H=0.1 \mathrm{~T}$ [Fig. 2(a)], and the $M(H)$ curve only linearly increases with $H$ when $H$ is applied along the $b$ direction (Fig. 3). These experimental results indicate a strong Ising anisotropy of the Mo moments with the easy axis along the $c$ direction in $\mathrm{La}_{5} \mathrm{Mo}_{4} \mathrm{O}_{16}$.

Figure 4(a) shows the $T$ dependence of the resistivity measured by the four-point probes method along the $a, b$, and $c$ axes ( $\rho_{a}, \rho_{b}$, and $\rho_{c}$, respectively), together with that measured by the two-point probes method along the $c$ axis. As can 

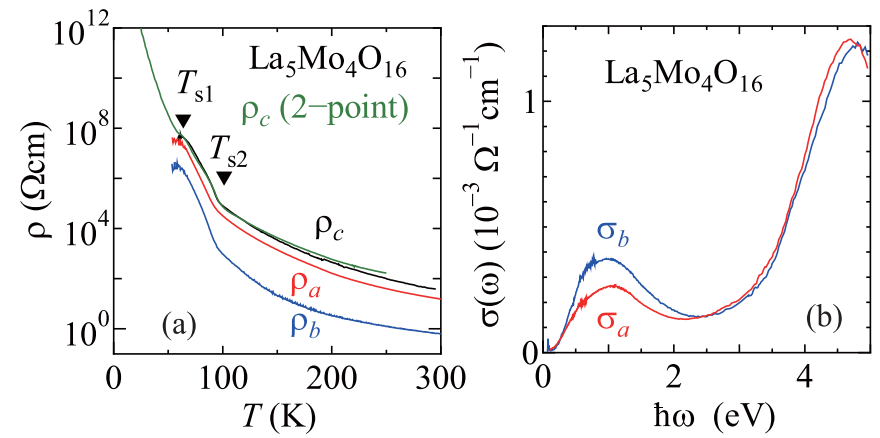

FIG. 4. (a) $T$ dependence of resistivity for $\mathrm{La}_{5} \mathrm{Mo}_{4} \mathrm{O}_{16}$ along the $a, b$, and $c$ axes measured by the four-point probes method and along the $c$ axis measured by the two-point probes method. (b) Optical conductivity spectra for $\mathrm{La}_{5} \mathrm{Mo}_{4} \mathrm{O}_{16}$ along the $a$ and $b$ axes derived from the optical reflectivity spectra.

be seen, $\rho$ in the three directions behave with $T$ similarly, although there is anisotropy in the absolute values of the resistivity, $\rho_{c}>\rho_{a}>\rho_{b}$. The anisotropy of the resistivity between the direction of the electric current along the $a b$ plane and that along the $c$ axis (Ref. [21]) can be reasonably explained by its two-dimensional crystal structure. To study the in-plane anisotropy in more detail, we measured the polarized optical reflectivity on the $a b$ plane. Figure 4(b) shows the optical conductivity spectra, $\sigma_{a}(\omega)$ and $\sigma_{b}(\omega)$, at room temperature derived from the Kramers-Kronig transformation of the reflectivity spectra. There are two peaks in both spectra at $\sim 1$ and $\sim 5 \mathrm{eV}$, corresponding to the Mott excitation from a $4 d$ state at a Mo ion to a $4 d$ state in the neighboring Mo ion, and the charge-transfer excitation from an oxygen $2 p$ state to a Mo $4 d$ state, respectively. The spectral weight of the peak at $\sim 1 \mathrm{eV}$ (corresponding to the Mott excitation) is approximately 1.5 times larger for the $\sigma_{b}(\omega)$ spectrum than for the $\sigma_{a}(\omega)$ spectrum. This anisotropy in the optical conductivity is consistent

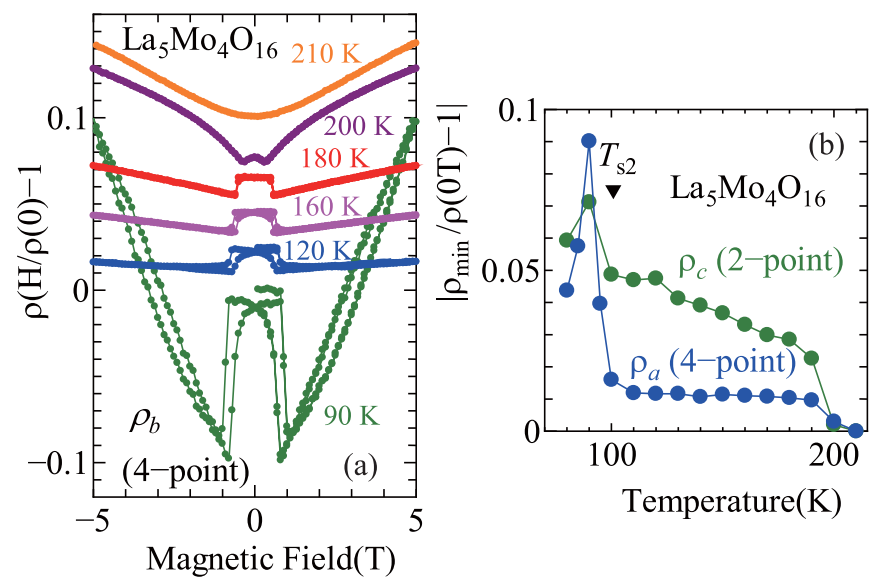

FIG. 5. (a) $\rho(H) / \rho(0)-1$, where $\rho(H)$ is the resistivity measured by the four-point probes method along the $b$ axis with the applied $H \| c$, as a function of $H$. (b) Magnitude of negative magnetoresistance, which is defined as $\left|\rho(0 \mathrm{~T}) / \rho_{\min }-1\right|$, where $\rho(0 \mathrm{~T})$ is the resistivity at $H=0$ and $\rho_{\min }$ is the minimum value of the resistivity when $H$ is increased from 0 to $5 \mathrm{~T}$, as a function of $T$ for $\rho_{b}$ shown in (a) and $\rho_{c}$ measured by a two-point probes method. (a)
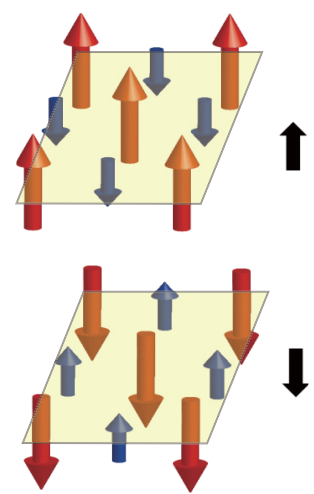

(b)
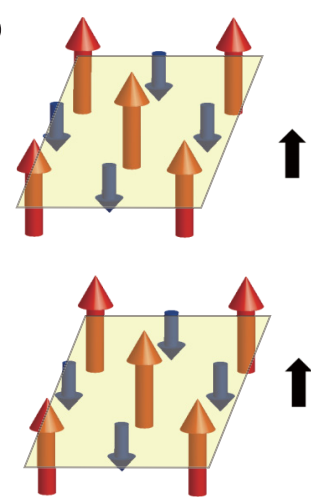

(c)
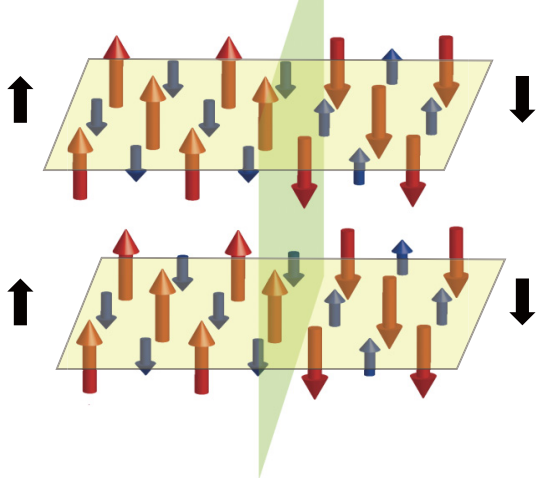

FIG. 6. [(a) and (b)] Schematic spin structures of the (a) interlayer antiferromagnetic (IL-AF) state, (b) interlayer ferromagnetic (IL-F) state, and (c) interlayer ferromagnetic state with magnetic domains.

with the anisotropy in the dc resistivity shown in Fig. 4(a), and is most likely caused by the different Mo-O-Mo angles in the $a$ and $b$ directions. Namely, the $\mathrm{MoO}_{6}$ octahedra rotate along the $b$ axis, as shown in Fig. 1, and thus, the Mo-O-Mo angle is $180^{\circ}$ along the $b$ axis, whereas it is away from $180^{\circ}$ along the $a$ axis, resulting in a larger transfer integral along the $b$ axis.

Figure 5(a) shows the $H(\| c)$ dependence of the resistivity measured by the four-point probes method along the $b$ axis at several temperatures $(\geqslant 90 \mathrm{~K})$. As can be seen, positive magnetoresistance proportional to $H^{2}$ is observed above $T_{N}=$ $200 \mathrm{~K}$, but below $T_{N}$, negative magnetoresistance, i.e., a sudden decrease in the resistivity with increasing $|H|$, occurs at $|H|<1 \mathrm{~T}$. This is the similar behavior to that observed in the resistivity along the $c$ axis by the two-point probes measurement (Refs. [19,21]), and is correlated with the metamagnetic transition shown in Fig. 3, namely, the change in the magnetic structure from the antiferromagnetic configuration of the ferrimagnetic moments between the layers [IL-AF state, Fig. 6(a)] to the ferromagnetic configuration [IL-F state, Fig. 6(b)].

Figure 5(b) shows the magnitude of the negative magnetoresistance, which is defined as $\left|\rho_{\min } / \rho(0 \mathrm{~T})-1\right|$, where $\rho(0 \mathrm{~T})$ is the resistivity at $H=0$ and $\rho_{\min }$ is the minimum value of the resistivity when $H$ is increased from 0 to $5 \mathrm{~T}$, as a function of $T$ for $\rho_{b}$ shown in Fig. 5(a) and for $\rho_{c}$ reported in Ref. [21]. As can be seen, the magnitude of the negative magnetoresistance for $\rho_{b}$ is several times smaller than that for 

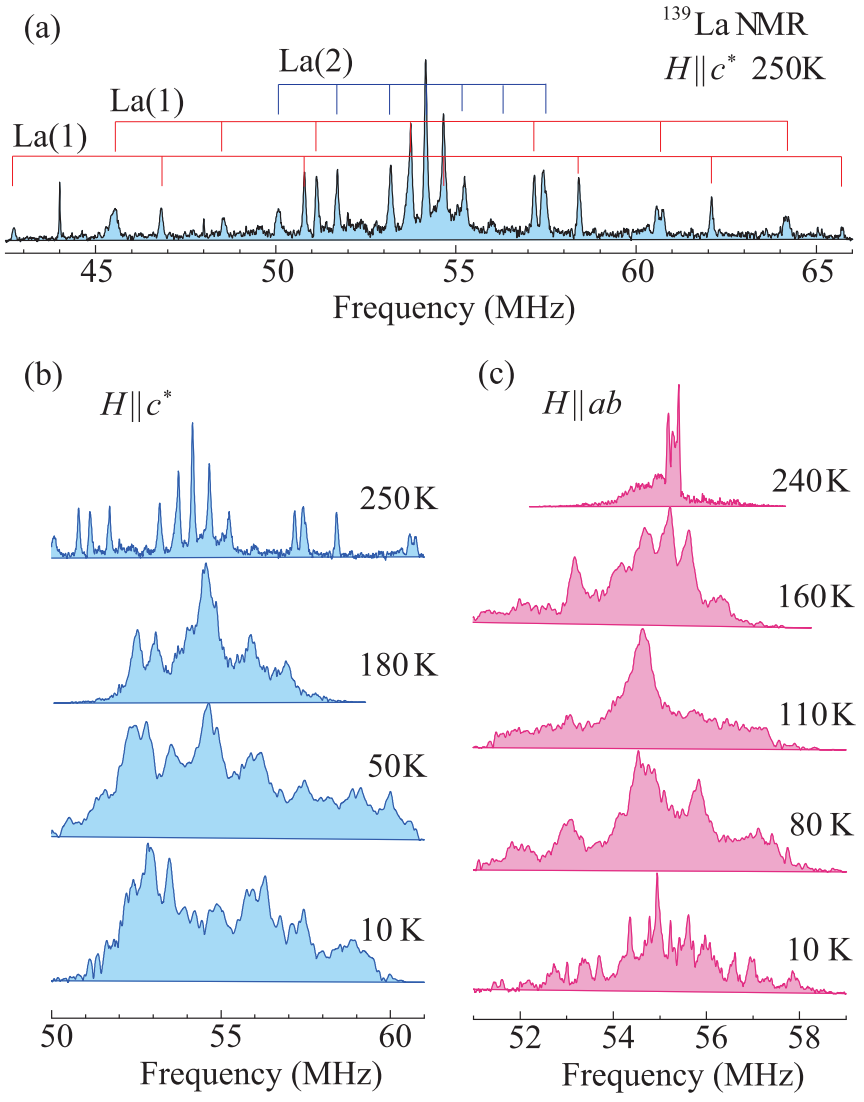

FIG. 7. ${ }^{139} \mathrm{La}$ NMR spectra in a single crystal of $\mathrm{La}_{5} \mathrm{Mo}_{4} \mathrm{O}_{16}$ under the constant $H$ of $9.089 \mathrm{~T}$ applied (a,b) normal to the $a b$ plane (here, we define $c^{*}$ as tilted by $5^{\circ}$ from the monoclinic $c$ axis), and (c) parallel to the $a b$ plane (the direction of $H$ within the $a b$ plane is unknown). The spectrum intensity is normalized at the maximum.

$\rho_{c}$ above $T_{s 2}=95 \mathrm{~K}$. However, below $T_{s 2}$ the magnitude of the negative magnetoresistance increases for both directions but particularly for $\rho_{b}$. This enhancement of the magnetoresistance immediately below $T_{s 2}$ may be correlated with the increase in the resistivity itself below $T_{s 2}$ shown in Fig. 4(a), which is caused by the orbital ordering of the Mo $4 d$ states, as discussed below.

\section{PHYSICAL PROPERTIES BELOW $T_{s 2}$ AND $T_{s 1}$}

As discussed in the introduction, anomalies appear in various properties at $T_{s 2}=95 \mathrm{~K}$ and $T_{s 1}=60 \mathrm{~K}$. For example, $\rho(T)$ exhibits anomalies, as shown in Fig. 4(a). In addition, a finite magnetization appears below $T_{s 1}$ at $H=0 \mathrm{~T}$ after applying $H \| c$ of larger than $1 \mathrm{~T}$, as shown in Figs. 2(d) and 3, indicating the ferromagnetic alignment of the ferrimagnetic moment between the layers, i.e., the IL-F phase shown in Fig. 6(b). However, this measurement of the uniform magnetization cannot distinguish whether the initial magnetic state (before $H$ is applied) below $T_{s 1}$ is the IL-F phase or not.

To investigate the magnetic state from a microscopic viewpoint, we performed NMR measurements. Figures 7(a) and 7(b) show the NMR spectra for the ${ }^{139}$ La nuclei at $H$ $=9.089 \mathrm{~T}$ along the $c^{*}$ axis (perpendicular to the $a b$ plane). The spectrum at $250 \mathrm{~K}$ [Fig. 7(a)] includes at least 14 lines
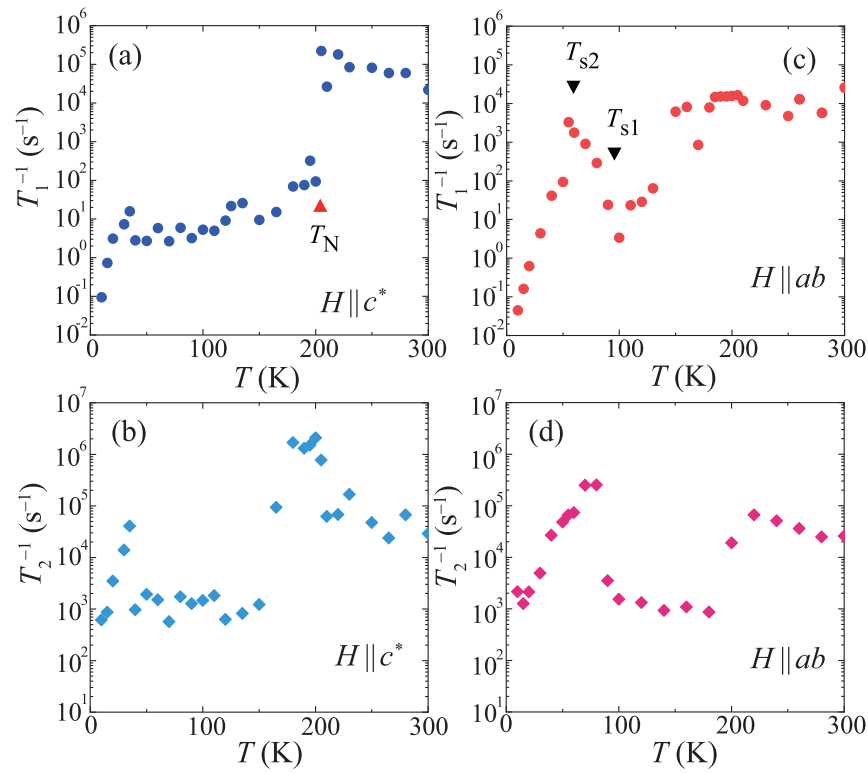

FIG. 8. [(a) and (c)] Nuclear spin-lattice relaxation rate $T_{1}^{-1}$ and (b, d) spin-echo decay rate $T_{2}^{-1}$ under $H$ normal and parallel to the $a b$ plane.

due to two La sites with the nuclear quadrupole splitting of the nuclear $\operatorname{spin}{ }^{139} I=7 / 2$. One La site, $\mathrm{La}(1)$, is close to the $\mathrm{Mo}^{4+}$ and $\mathrm{Mo}^{5+}$ sites on the square lattice showing magnetic ordering, whereas the other La site, $\mathrm{La}(2)$, is located near nonmagnetic $\mathrm{Mo}$ sites in the $\mathrm{Mo}_{2} \mathrm{O}_{10}$ pillar (Fig. 1). Under the magnetic field deviated from the mirror $a c$ plane, the $\mathrm{La}(1)$ site in the unit cell (monoclinic $C 2 / \mathrm{m}$ ) is divided into two groups whereas $\mathrm{La}(2)$ located at the symmetrical position is not. As a result, we observed three sets of nuclear quadrupole splitting for $H$ along the $c^{*}$ axis [Fig. 7(a)]. Below $T_{\mathrm{N}}=200 \mathrm{~K}$, the NMR spectrum broadens as the local field develops. The spectrum further broadens below $50 \mathrm{~K}$. The residual inhomogeneous broadening indicates that the local fields from the Mo moments do not completely cancel at the La sites. Figure 7(c) shows the NMR spectra for $H \| a b$ (the direction of $H$ within the $a b$ plane is unknown). At $240 \mathrm{~K}$, the spectrum for $\mathrm{La}(2)$ gives sharp lines whereas that for $\mathrm{La}$ (1) gives broad spectra. After showing the broadening in the intermediate $T$ range, a number of sharp lines appear at $10 \mathrm{~K}$.

Figure 8 shows $T_{1}^{-1}$ and $T_{2}^{-1}$, which respectively measure transverse and longitudinal spin fluctuations against external $H$. For $H \| c^{*}$, both $T_{1}^{-1}$ and $T_{2}^{-1}$ are slightly enhanced above $T_{\mathrm{N}}$ owing to the slowing down of spin fluctuations and steeply decrease below $T_{\mathrm{N}}$. Reflecting the Ising-like anisotropy, $T_{2}^{-1}$ detecting fluctuations along $c^{*}$ is more enhanced than $T_{1}^{-1}$. No anomaly is observed down to low $T$ across $T_{\mathrm{s} 1}$ and $T_{\mathrm{s} 2}$ in $T_{1}^{-1}$ and $T_{2}^{-1}$. This is consistent with the result of the static magnetization measurement that all the ferrimagnetic moments in each layer are polarized to the $c^{*}$ direction at $9 \mathrm{~T}$ below $T_{\mathrm{N}}$. On the other hand, when $H$ is applied within the $a b$ plane, $T_{1}^{-1}$ and $T_{2}^{-1}$ are enhanced near $T_{s 2}=95 \mathrm{~K}$ and suppressed below $T_{s 1}=60 \mathrm{~K}$. This is clear evidence that a magnetic phase transition occurs at $T_{s 1}$ when there is no $H$ along the $c$ axis. In other words, the NMR result indicates that the magnetic state below $T_{s 1}$ without $H$ is no 
(a)

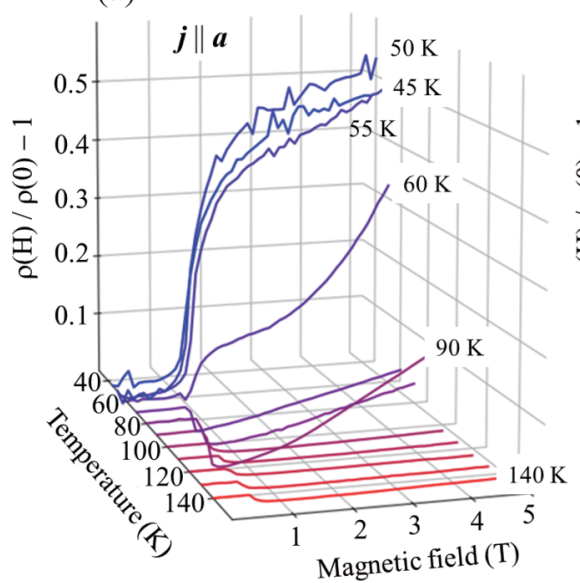

(b)

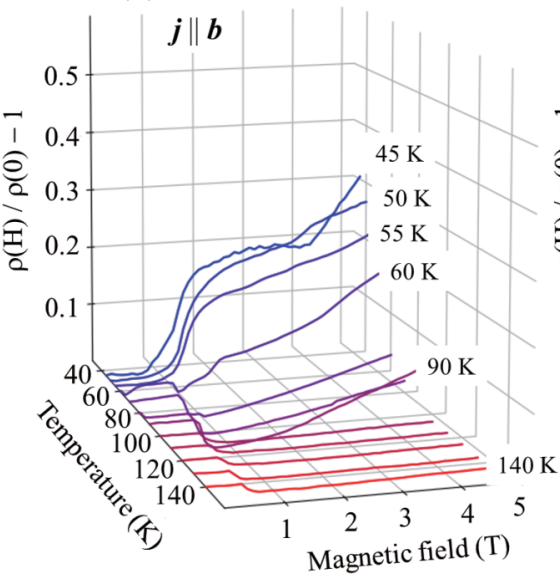

(c)

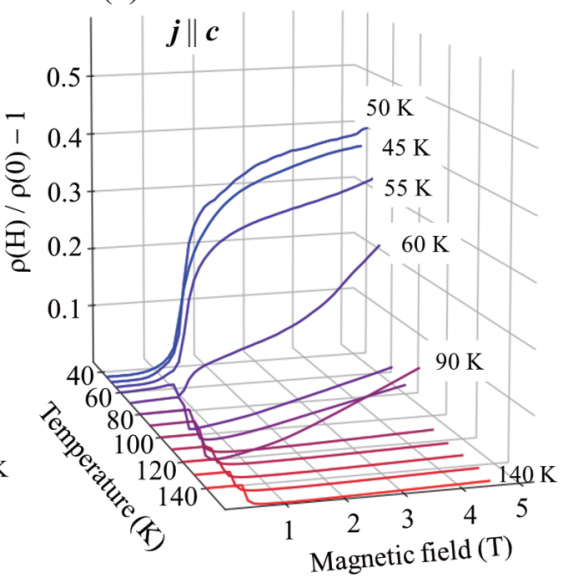

FIG. 9. [(a)-(c)] $\rho(H) / \rho(0)-1$, where $\rho(H)$ is the resistivity measured by a two-point probes method along the (a) $a$, (b) $b$, and (c) $c$ axes with the applied $H \| c$, as a function of $H$ at various temperatures.

more an interlayer antiferromagnetic (IL-AF) state [as shown in Fig. 6(a)], but an interlayer ferromagnetic (IL-F) state [as shown in Fig. 6(b)].

Figures 9(a)-9(c) show the resistivity $\rho$ along the $a, b$, and $c$ axes, respectively, as a function of $H \| c$ from 0 to $5 \mathrm{~T}$ in the initial magnetization process. The behavior of $\rho$ after the initial magnetization process is shown in Fig. 10(a) along the $b$ axis at $50 \mathrm{~K}$ and $90 \mathrm{~K}$ as an example. For all the directions, $\rho$ behaves similarly; namely, above $70 \mathrm{~K}, \rho$ sharply decreases with increasing $H$ at $\sim 1 \mathrm{~T}$, corresponding to the negative magnetoresistance associated with the metamagnetic transition from the IL-AF state to the IL-F state as discussed above. However, a gradual increase in $\rho$ appears below $70 \mathrm{~K}$, and the magnitude of such positive magnetoresistance increases with decreasing $T$ and is saturated at $\sim 50 \mathrm{~K}$. As shown in Fig. 10(a), $\rho_{b}$ (along the plane) does not decrease but remains large values with a further sweep of $H$ between 5 and $-5 \mathrm{~T}$, similarly to the behavior of $\rho_{c}$ previously reported [21]. Figure 10(b) shows the $T$ dependence of the positive magnetoresistance (the left axis) defined

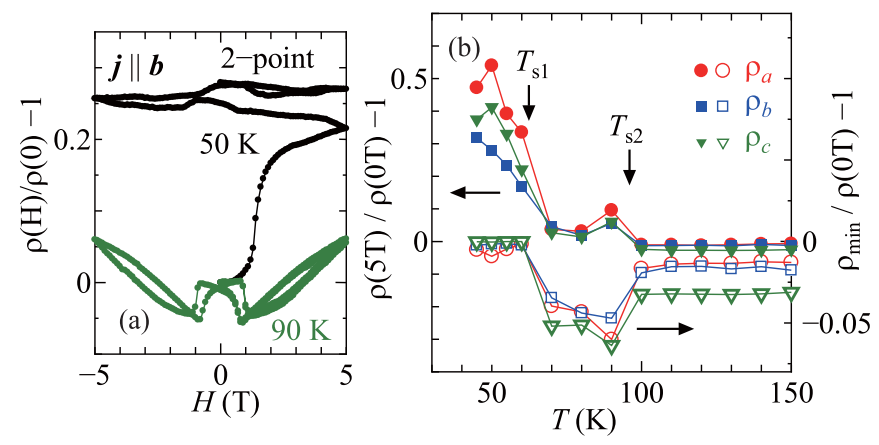

FIG. 10. (a) $\rho(H) / \rho(0)-1$, where $\rho(H)$ is the resistivity measured by a two-point probes method along the $b$, with sweeping $H \| c$ for $-5 \leqslant H \leqslant 5 \mathrm{~T}$ at 50 and $90 \mathrm{~K}$. (b) $T$ dependence of the positive magnetoresistance (left axis) defined as $\rho(5 \mathrm{~T}) / \rho(0 \mathrm{~T})-1$, where $\rho(5 \mathrm{~T})$ and $\rho(0 \mathrm{~T})$ are the resistivities at $5 \mathrm{~T}$ and $0 \mathrm{~T}$, respectively, and the negative magnetoresistance (right axis) defined as $\rho_{\min } / \rho(0 \mathrm{~T})-1$, where $\rho_{\min }$ is the minimum value of the resistivity when $H$ is increased from 0 to $5 \mathrm{~T}$. as $\rho(5 \mathrm{~T}) / \rho(0 \mathrm{~T})-1$, where $\rho(5 \mathrm{~T})$ and $\rho(0 \mathrm{~T})$ are the resistivities at 5 and $0 \mathrm{~T}$, respectively, together with the negative magnetoresistance (the right axis) defined as $\rho_{\min } / \rho(0 \mathrm{~T})-1$, where $\rho_{\min }$ is the minimum value of the resistivity when $H$ is increased from 0 to $5 \mathrm{~T}$. The absolute value of the negative magnetoresistance increases below $T_{s 2}=95 \mathrm{~K}$, but decreases again below $T_{s 1}=60 \mathrm{~K}$, at which the positive magnetoresistance $(\rho(5 \mathrm{~T}) / \rho(0 \mathrm{~T})-1)$ increases. Although a nonvolatile positive magnetoresistance is observed for all the directions of the electric current, there is a small anisotropy, $\Delta \rho_{a} \sim \Delta \rho_{c}>$ $\Delta \rho_{b}$.

To understand the characteristics of $T_{s 1}$ and $T_{s 2}$ from the structural viewpoint, we measured the strain of the sample. Figure 11(a) shows the $T$ dependence of strain along the three axes, $(\Delta L / L)_{a},(\Delta L / L)_{b}$, and $(\Delta L / L)_{c}$. In addition to the small anomaly at $T_{N},(\Delta L / L)_{b}$ decreases at $T_{s 2}$ but increases at $T_{s 1}$, whereas $(\Delta L / L)_{c}$ increases at $T_{s 2}$ but decreases at $T_{s 1}$ with decreasing $T$. $(\Delta L / L)_{a}$ barely exhibits anomalies at these temperatures. This clearly indicates that the anomalies at $T_{s 1}$ and $T_{s 2}$ are associated with structural anomalies.

We also measured the strain with applied $H$. As shown in Fig. 11(b), $(\Delta L / L)_{c}$ barely changes with $H \| c$, except at $T$

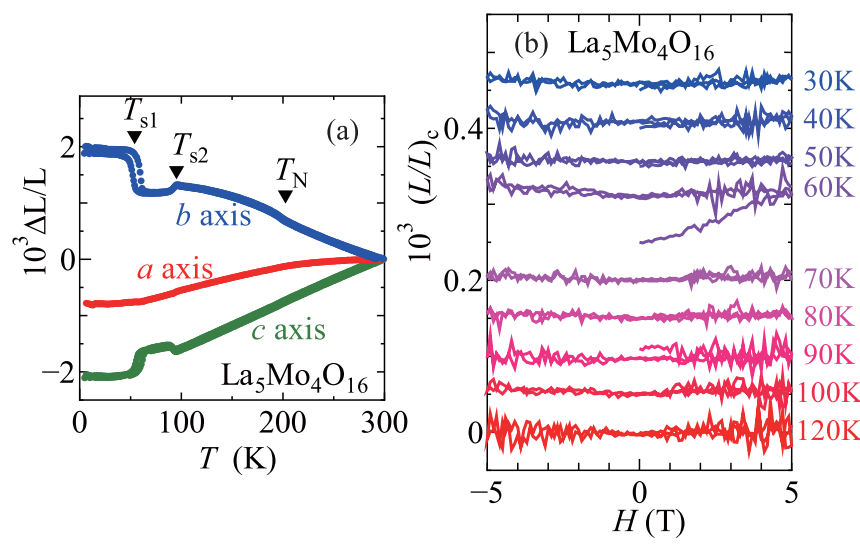

FIG. 11. (a) Strain $\Delta L / L$ with the zero point at $300 \mathrm{~K}$ along the $a, b$, and $c$ axes. (b) Strain $\Delta L / L$ along the $c$ axis as a function of $H \| c$ at various temperatures. The data are offset for clarity. 


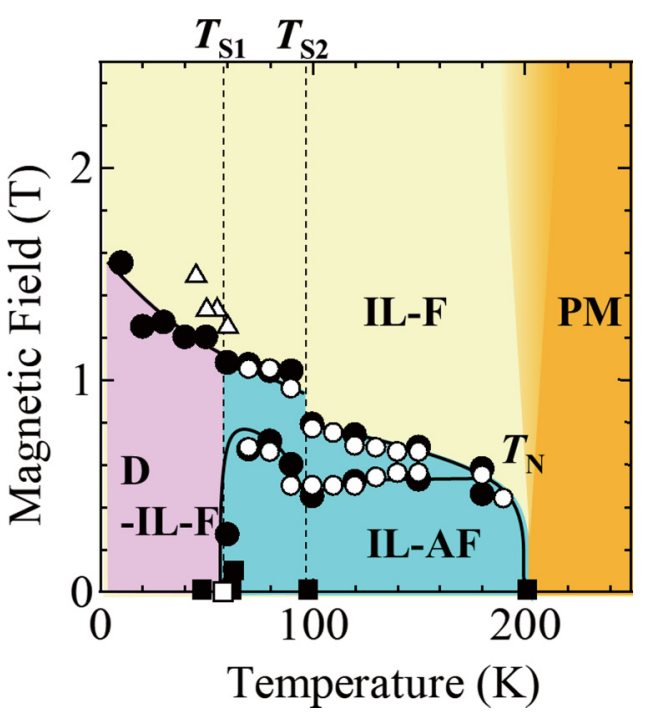

FIG. 12. Magnetic phase diagram of $\mathrm{La}_{5} \mathrm{Mo}_{4} \mathrm{O}_{16}$ in the space of $T$ and $H$ along the $c$ axis. The data from the magnetization measurement are shown by solid symbols, whereas those of resistivity are shown by open symbols. Squares are from the $T$ dependence of either the magnetization (Fig. 2) or resistivity (Fig. 4(a)). Circles are from the $H$ dependence of the magnetization (Fig. 3) or negative magnetoresistance (Fig. 5). Triangles are from the $H$ dependence of the positive magnetoresistance (Fig. 9).

close to $T_{s 1}$, where $(\Delta L / L)_{c}$ slightly increases. This means that $T_{s 1}$ and $T_{s 2}$ are robust against the magnetic field. The small anomaly with $H$ near $T_{s 1}$ is due to the fact that the magnetic state between $T_{s 1}$ and $T_{s 2}$ has a larger magnetic moment than the other $T$ range at large $H$, as shown in Fig. 2(d), and thus, the magnetic state between $T_{s 1}$ and $T_{s 2}$ is favored with applied $H$, resulting in a small shift of $T_{s 1}$ to a lower $T$ with $H$.

The experimental results above are summarized as a phase diagram in Fig. 12. The phase below $T_{s 1}=60 \mathrm{~K}$ at low $H$ is named as a "D-IL-F" (disordered interlayer-ferromagnetic) phase, since as already reported [21], the relaxation behavior that is likely to arise from disorder have been observed in this region. We discuss this issue in more details in the next section.

\section{MAGNETIC FORCE MICROSCOPY AND THE MEMORY EFFECT IN THE MAGNETIZATION}

To understand the magnetic state below $T_{s 1}$ (the D-IL-F phase in Fig. 12), we measured the MFM image [12,28] on the $a b$ plane of $\mathrm{La}_{5} \mathrm{Mo}_{4} \mathrm{O}_{16}$ at various temperatures without a magnetic field, as shown in Fig. 13. Above $70 \mathrm{~K}$, magnetic domains are barely observed. This is consistent with the fact that this compound is in the IL-AF phase with no spontaneous magnetization above $T_{s 1}=60 \mathrm{~K}$. On the other hand, below $70 \mathrm{~K}$, magnetic domains of several hundred $\mathrm{nm}$ in length appear, whose size decreases to $\sim 100 \mathrm{~nm}$ at $T=50$ and $40 \mathrm{~K}$. This result indicates that the ground state changes from the IL-AF state to the IL-F state with a spontaneous magnetization. With further decreasing $T$, however, such magnetic domains become blurred again probably because the size of the magnetic domains becomes larger than the imaging area.

We also observed the magnetic domains at $50 \mathrm{~K}$ with the applied $H$ along the $c$ axis by MFM, as shown in Fig. 14. The magnetic domains are almost smeared out at $H=0.3 \mathrm{~T}$, and with further increasing $H$, reducing $H$, or increasing $|H|$ with the opposite sign, the magnetic domains as observed before $H$ is applied do not appear again.

It should be emphasized that such magnetic domains have a disordered structure. The existence of disorder in the magnetic state below $T_{s 1}$ has been suggested in the previous study by the relaxation behavior for wide ranges of both $H$ and $T$ [21]. In the present study, the so-called memory effect in the magnetization below $T_{s 1}$ has been investigated. Namely, the sample is cooled without applied $H$ at a constant cooling rate (=30 K/min in this experiment) to a certain $T=T_{H}$, and $T$ is halted there over a certain time (one hour in this experiment), and the cooling is restarted at the same cooling rate down to the lowest $T$ ( $=5 \mathrm{~K}$ ). After that, $H=0.1 \mathrm{~T}$ along the $c$ axis is applied and the magnetization $M$ is measured with increasing $T$ at a constant warming rate $(=10 \mathrm{~K} / \mathrm{min}$ in this experiment).

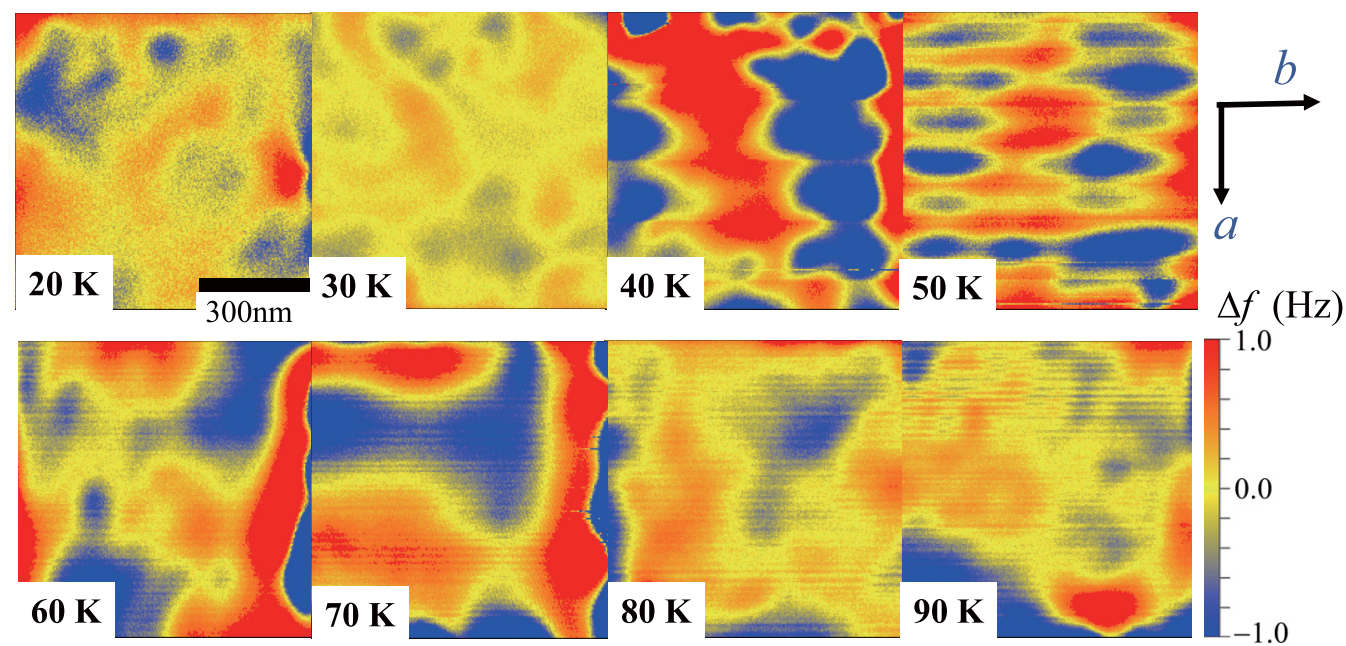

FIG. 13. Magnetic-force microscopy (MFM) images taken along the $a b$ plane of $\mathrm{La}_{5} \mathrm{Mo}_{4} \mathrm{O}_{16}$ at various temperatures. The size of each image is $800 \mathrm{~nm} \times 800 \mathrm{~nm}$. 


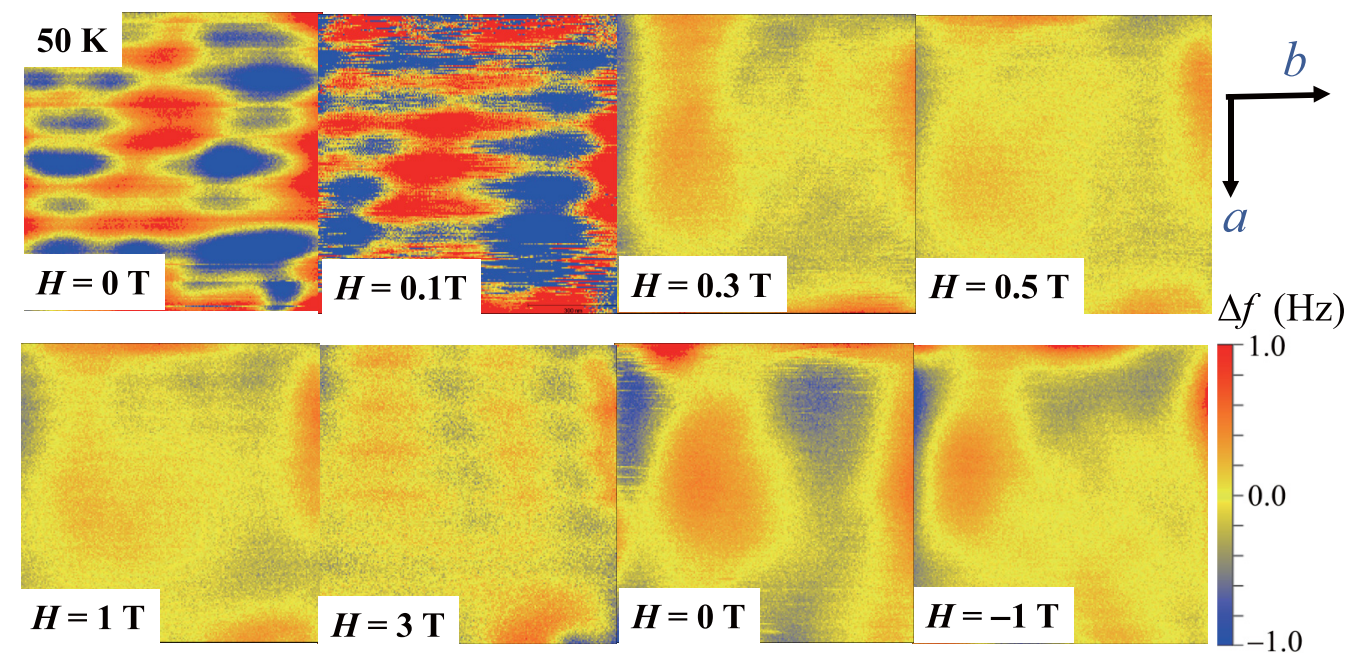

FIG. 14. Magnetic-force microscopy (MFM) images taken along the $a b$ plane of $\mathrm{La}_{5} \mathrm{Mo}_{4} \mathrm{O}_{16}$ at $50 \mathrm{~K}$ at various $H$ along the $c$ axis. The size of each image is $800 \mathrm{~nm} \times 800 \mathrm{~nm}$.

The curves of $M(T)$ are plotted for various values of $T_{H}$ in Figs. 15(a) and 15(b). As can be seen, $M(T)$ starts increasing with increasing $T$ at a certain $T=T_{\min }$, which is close to $T_{H}$, as shown in Fig. 15(c).

Memory effect in the magnetization is often observed experimentally in spin-glass compounds [29], which is caused by the multivalley structure of the energy landscape arising from the disorder in the systems. Similar behavior was observed also in magnetic nanoparticles [30,31], where the

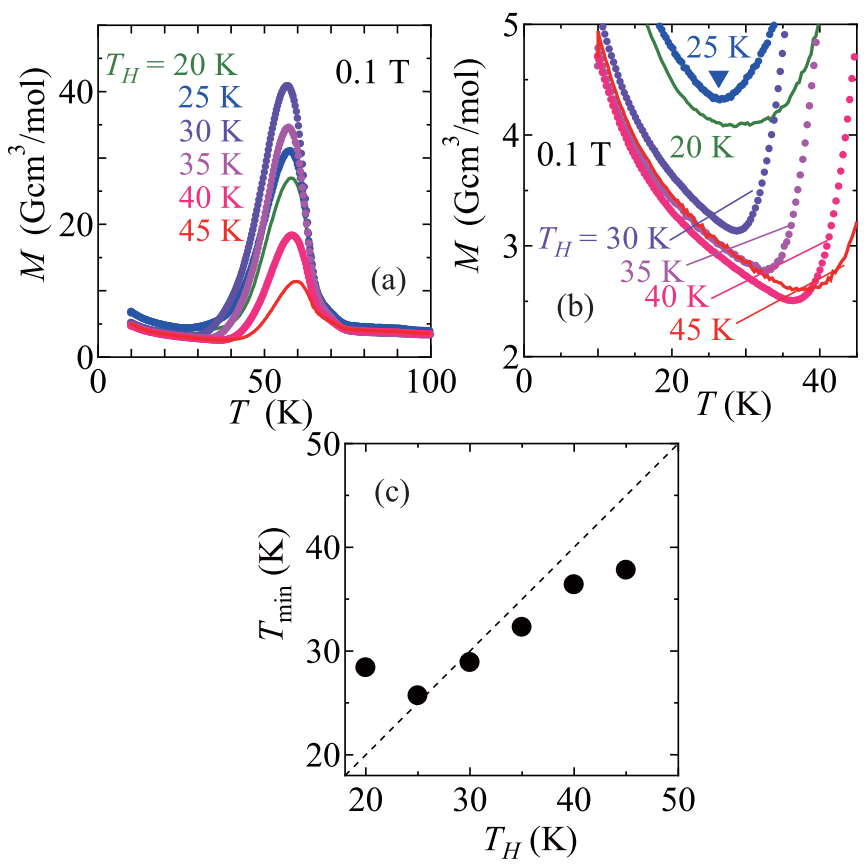

FIG. 15. [(a) and (b)] Magnetization in a warming run with $H=$ $0.1 \mathrm{~T}$ along the $c$ axis after the sample is cooled (without $H$ ) to $T=$ $T_{H}$ at a cooling rate of $30 \mathrm{~K} / \mathrm{min}$, then the $T$ is held at $T_{H}$ for $1 \mathrm{~h}$, and then, cooling is continued again to $5 \mathrm{~K}$. (b) is an expanded figure of (a). The triangle in (b) is $T_{\min }$ for $25 \mathrm{~K}$. (c) $T_{\min }$, which is defined as $T$ at which the magnetization becomes minimum with increasing $T$ as shown in (b), as a function of $T_{H}$. existence of the $T$-dependent distribution of the relaxation time arising from the distribution of the particle size plays an important role $[32,33]$. The present experimental result indicates that the magnetic state below $T_{s 1}$ involves a significant disorder despite its crystalline form without any substitution, and it is likely caused by the disordered magnetic-domain structures shown in Figs. 13 and 14.

The appearance and disappearance of the magnetic domains with $T$ and $H$ shown in Figs. 13 and 14 seem to be correlated with the nonvolatile positive magnetoresistance of the present compound, indicating the electrical conduction along the domain walls between the upward and downward magnetizations. Namely, even though $\mathrm{La}_{5} \mathrm{Mo}_{4} \mathrm{O}_{16}$ is insulating as a bulk property, it is assumed that the boundary between the domains with upward and downward magnetizations (domain walls) is conducting. As exemplified in the MFM image shown in Fig. 13, ferromagnetic domains of $\sim 100 \mathrm{~nm}$ size appear below $T_{s 1}=60 \mathrm{~K}$, and if the electric current can flow along the boundaries, the resistivity will decrease with the formation of the ferromagnetic domains. This corresponds to the anomaly in $\rho(T)$ at $T_{s 1}$ shown in Fig. 4(a) and is schematically represented by the bottom arrow in Fig. 16. With the applied $H$ along the $c$ axis, these magnetic domains disappear, resulting in the disappearance of the electrical conduction along the domain walls, which causes positive magnetoresistance, as shown in Fig. 9. This process is schematically represented in Fig. 16 by the left arrow. As exemplified in the MFM image, once the magnetic domains of $\sim 100 \mathrm{~nm}$ size disappear, they never appear again with further sweeping of $H$, as shown in Fig. 14. This means that the magnetic domains that appear when the magnetization in the IL-F phase is flipped with $H$ are much larger than $100 \mathrm{~nm}$. This is also consistent with the nonvolatile behavior of the positive magnetoresistance; namely, the resistivity does not decrease but remains large values with a further sweep of $H$ because of the small number of magnetic domain walls.

There are two issues that should be addressed. First, the magnetic domains in Fig. 14 seem to disappear at $H=$ $0.3 \mathrm{~T}$, but positive magnetoresistance appears up to $\sim 1 \mathrm{~T}$, as shown in Fig. 9. Since this compound exhibits relaxation 


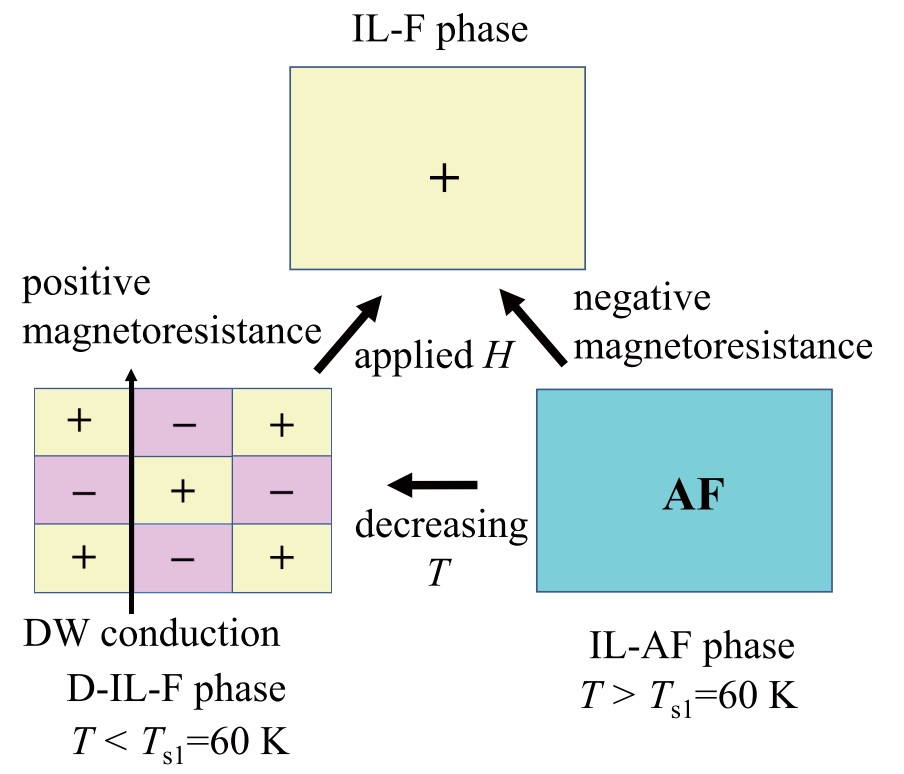

FIG. 16. Schematic pictures of the positive magnetoresistance below $T_{s 1}$ and negative magnetoresistance above $T_{s 1}$ in $\mathrm{La}_{5} \mathrm{Mo}_{4} \mathrm{O}_{16}$.

behavior, namely, a time dependence in the magnetization and resistivity [21], it is difficult to compare the results by the different experiments with different time scales quantitatively. Furthermore, the existence of the memory effect suggests that there is a distribution in the size of the magnetic domains below $T_{s 1}$ in this compound. It is likely that larger magnetic domains disappear at higher $H$, contributing to the magnetoresistance at higher $H$.

Second, the magnetic domains should exist also in the IL$\mathrm{AF}$ phase, though no positive magnetoresistance is observed experimentally in the IL-AF phase. As will be discussed in Sec. VI, the magnetic phase transition from the IL-AF phase to the IL-F phase is induced by the orbital ordering and the sign change in the interlayer magnetic interaction in its consequence. Such a sudden change in the magnetic interaction far below $T_{N}=200 \mathrm{~K}$ results in the nonequilibrium character in the magnetic domains and the relatively small size of the magnetic domains $(\sim 100 \mathrm{~nm})$ in the IL-F phase in spite of its large Ising anisotropy. On the other hand, the magnetic domains in the IL-AF gradually evolves below $T_{N}$ and thus, there is no reason that the size of the magnetic domains is such small. This difference can lead to the different magnitude in the positive magnetoresistance between the IL-F phase and the IL-AF phase. We will discuss this issue again in Sec. VII.

\section{HARTREE-FOCK CALCULATION}

To theoretically clarify the origin of the phase transitions at $T_{s 1}$ and $T_{s 2}$, and to prove the emergence of conducting domain walls in $\mathrm{La}_{5} \mathrm{Mo}_{4} \mathrm{O}_{16}$, we perform the model Hartree-Fock calculations [34]. We employ a multiorbital Hubbard model to describe the Mo $4 d$ electrons in $\mathrm{La}_{5} \mathrm{Mo}_{4} \mathrm{O}_{16}$, which is given by

$$
\begin{aligned}
\mathcal{H}= & \sum_{i, j} \sum_{\mu, \nu} \sum_{\sigma} t_{i, \mu ; j, v} c_{i \mu \sigma}^{\dagger} c_{j v \sigma}+\sum_{i} \sum_{\mu, \nu} \sum_{\sigma, \sigma^{\prime}} \lambda_{\mu \sigma ; \nu \sigma^{\prime}} c_{i \mu \sigma}^{\dagger} c_{i v \sigma^{\prime}}+\Delta_{\mathrm{st}} \sum_{i \in \mathrm{Mo}^{4+}} \sum_{\mu} c_{i \mu \sigma}^{\dagger} c_{i \mu \sigma}+\Delta_{x y} \sum_{i} \sum_{\mu \in x y} c_{i \mu \sigma}^{\dagger} c_{i \mu \sigma} \\
& +H_{\mathrm{ext}} \sum_{i} \sum_{\mu}\left(c_{i \mu \uparrow}^{\dagger} c_{i \mu \uparrow}-c_{i \mu \downarrow}^{\dagger} c_{i \mu \downarrow}\right)+u \sum_{i} \sum_{\mu} c_{i \mu \uparrow}^{\dagger} c_{i \mu \uparrow} c_{i \mu \downarrow}^{\dagger} c_{i \mu \downarrow}+u^{\prime} \sum_{i} \sum_{\mu \neq \nu} c_{i \mu \uparrow}^{\dagger} c_{i \mu \uparrow} c_{i \nu \downarrow}^{\dagger} c_{i \nu \downarrow} \\
& +\left(u^{\prime}-j\right) \sum_{i} \sum_{\mu>\nu} \sum_{\sigma} c_{i \mu \sigma}^{\dagger} c_{i \mu \sigma} c_{i v \sigma}^{\dagger} c_{i v \sigma}+j^{\prime} \sum_{i} \sum_{\mu \neq \nu} c_{i \mu \uparrow}^{\dagger} c_{i \nu \uparrow} c_{i \nu \downarrow}^{\dagger} c_{i \mu \downarrow}+j \sum_{i} \sum_{\mu \neq \nu} c_{i \mu \uparrow}^{\dagger} c_{i \nu \uparrow} c_{i \mu \downarrow}^{\dagger} c_{i \nu \downarrow},
\end{aligned}
$$

where the indices $i$ and $j$ denote the Mo sites, whereas the indices $\mu$ and $v$ denote threefold Mo $t_{2 g}$ orbitals $\left(4 d_{x y}, 4 d_{y z}\right.$, $\left.4 d_{z x}\right)$. In this model, the Mo $e_{g}$ orbitals $\left(4 d_{x^{2}-y^{2}}, 4 d_{3 z^{2}-r^{2}}\right)$ are not taken into account because the crystal-field splitting between the $t_{2 g}$ and $e_{g}$ levels is so large that the Mo $e_{g}$ orbitals are always unoccupied.

The first term describes the indirect hoppings of Mo $3 d$ electrons between adjacent Mo $t_{2 g}$ orbitals mediated by an inbetween $\mathrm{O} 2 p$ orbital. Here, we assume an undistorted square lattice of corner-shared $\mathrm{MoO}_{6}$ octahedra in the $a b$ plane and neglect their tilting distortion. The resulting high symmetry of the crystal structure restricts the indirect electron hoppings among the Mo $t_{2 g}$ orbitals in the $a b$ plane. More specifically, only the following transfer integrals $t_{i, \mu ; j, v}$, which come from a couple of $p d \pi$ hybridizations between Mo $t_{2 g}$ and $\mathrm{O} 2 p$ orbitals, have nonzero values, all of which are represented by the same parameter $t_{a b}$ :

$$
t_{a b} \equiv t_{i, x y ; i \pm \hat{\boldsymbol{x}}, x y}=t_{i, z x ; i \pm \hat{\boldsymbol{x}}, z x}=t_{i, x y ; i \pm \hat{\mathbf{y}}, x y}=t_{i, y z ; i \pm \hat{\mathbf{y}}, y z} .
$$

The square lattices of $\mathrm{MoO}_{6}$ octahedra are stacked along the $c$ axis and connected loosely by the nonmagnetic $\mathrm{Mo}_{2} \mathrm{O}_{10}$ pillar or the $\mathrm{La}^{3+}$ cation. We consider the following interlayer transfer integrals:

$$
t_{c} \equiv t_{i, y z ; i \pm \hat{z}, y z}=t_{i, z x ; i \pm \hat{z}, z x} .
$$

The second term describes the spin-orbit interaction in the Mo $t_{2 g}$ orbitals. Using the basis of $\left\{\left|d_{i, x y} \uparrow\right\rangle,\left|d_{i, x y} \downarrow\right\rangle,\left|d_{i, y z} \uparrow\right\rangle,\left|d_{i, y z} \downarrow\right\rangle,\left|d_{i, z x} \uparrow\right\rangle,\left|d_{i, z x} \downarrow\right\rangle\right\}$, this term is given in matrix form as

$$
\mathcal{H}_{\mathrm{SOI}}=\left(\begin{array}{cccccc}
0 & 0 & 0 & \frac{\zeta}{2} & 0 & -i \frac{\zeta}{2} \\
0 & 0 & -\frac{\zeta}{2} & 0 & -i \frac{\zeta}{2} & 0 \\
0 & -\frac{\zeta}{2} & 0 & 0 & i \frac{\zeta}{2} & 0 \\
\frac{\zeta}{2} & 0 & 0 & 0 & 0 & -i \frac{\zeta}{2} \\
0 & i \frac{\zeta}{2} & -i \frac{\zeta}{2} & 0 & 0 & 0 \\
i \frac{\zeta}{2} & 0 & 0 & i \frac{\zeta}{2} & 0 & 0
\end{array}\right),
$$


where $\zeta$ is the coupling constant. The third term describes staggered site potentials in the $a b$ plane. Consideration of this term is necessary to reproduce the experimentally observed alternate configuration of $\mathrm{Mo}^{4+}\left(d^{2}, S=1\right)$ and $\mathrm{Mo}^{5+}\left(d^{1}\right.$, $S=1 / 2$ ) ions. Its physical origin is the different crystallographic environments at the two Mo sites. Specifically, the $\mathrm{Mo}^{4+}$ sites are sandwiched by two $\mathrm{Mo}_{2} \mathrm{O}_{10}$ pillars, whereas the $\mathrm{Mo}^{5+}$ sites are sandwiched by the $\mathrm{La}^{3+}$ ions along the $c$ axis. We consider a higher potential energy of $\Delta_{\mathrm{st}}(>0)$ for the $\mathrm{Mo}^{5+}$ sites. The fourth term describes the $t_{2 g}$-level splitting due to the two-dimensional anisotropy, which lowers the energy of the $4 d_{x y}$ orbital by $\Delta_{x y}$ compared with those of the $4 d_{y z}$ and $4 d_{z x}$ orbitals at each Mo site. The fifth term describes the Zeeman interactions associated with an external magnetic field $\boldsymbol{H}_{\mathrm{ext}}=\left(0,0, H_{\mathrm{ext}}\right)$ applied along the $c(z)$ axis where the spin quantization axis is chosen to be the $c(z)$ axis in the present model.

The remaining terms represent the $d$ - $d$ Coulomb interactions, which are expressed by the Kanamori parameters $u, u^{\prime}$, $j$, and $j^{\prime}$ [35]. To keep the rotational invariance in the real space, we assume the relationships $u^{\prime}=u-2 j$ and $j=j^{\prime}$. The parameters also satisfy the relationship $U_{\mathrm{av}}=u-(20 / 9) j$, where $U_{\mathrm{av}}$ is the multiplet average of the $d-d$ Coulomb interactions.

We apply the unrestricted Hartree-Fock approximation to these Coulomb-interaction terms. Details will be described in Appendix. We used the following parameter values for the calculations: $t_{a b}=1 \mathrm{eV}, t_{c}=0.032 \mathrm{eV}, u=5 \mathrm{eV}, j=j^{\prime}=$ $0.625 \mathrm{eV}, u^{\prime}(=u-2 j)=3.75 \mathrm{eV}, \zeta=0.4 \mathrm{eV}, \Delta_{\mathrm{st}}=1 \mathrm{eV}$, and $\Delta_{x y}=-0.4 \mathrm{eV}$. We first mention that the $4 d_{x y}$ orbitals are lower in energy by $\Delta_{x y}$ and, thereby, are uniformly occupied at all $\mathrm{Mo}^{4+}$ and $\mathrm{Mo}^{5+}$ sites in this compound. Importantly, the $4 d^{2}$ state at each $\mathrm{Mo}^{4+}$ site has orbital degrees of freedom, that is, another electron can occupy either the $4 d_{y z}$ or $4 d_{z x}$ orbital to form a $S=1$ spin.

In the calculations for a system with $4 \times 4 \times 2$ Mo sites with periodic boundary conditions, we reproduced several orbital-spin phases caused by couplings between spin and orbital degrees of freedom and their phase transitions induced by the variation of $T$ and the application of $H$. When the external magnetic field is absent $\left(H_{\mathrm{ext}}=0\right)$, we observed two successive phase transitions with decreasing $T$. The system first enters from a paramagnetic phase at higher $T$ to an antiferromagnetic phase at intermediate $T$. In these two phases, the Mo $4 d$ orbitals are not ordered (an orbital disordered state), and the $4 d_{y z}$ and $4 d_{z x}$ orbitals are equally occupied at every $\mathrm{Mo}^{4+}$ site. With further decreasing $T$, the system subsequently enters an orbital ordered phase. Through this orbital disorder-to-order transition, the interlayer spin coupling changes from antiferromagnetic to ferromagnetic.

This orbital ordering is characterized by a staggered arrangement of $4 d_{y z}$ and $4 d_{z x}$ orbitals at $\mathrm{Mo}^{4+}$ sites shown in Fig. 17(a). The staggered orbital configuration in the $a b$ plane is favored by the fourth-order perturbation processes with respect to the transfer integrals $t_{a b}$ mediated by in-between unoccupied $\mathrm{Mo}^{5+} t_{2 g}$ orbitals, whereas the interlayer staggered orbital configuration is favored by the second-order perturbation processes with respect to the transfer integrals $t_{c}$ [Figs. 17(b) and 17(c)]. The predicted orbital configuration

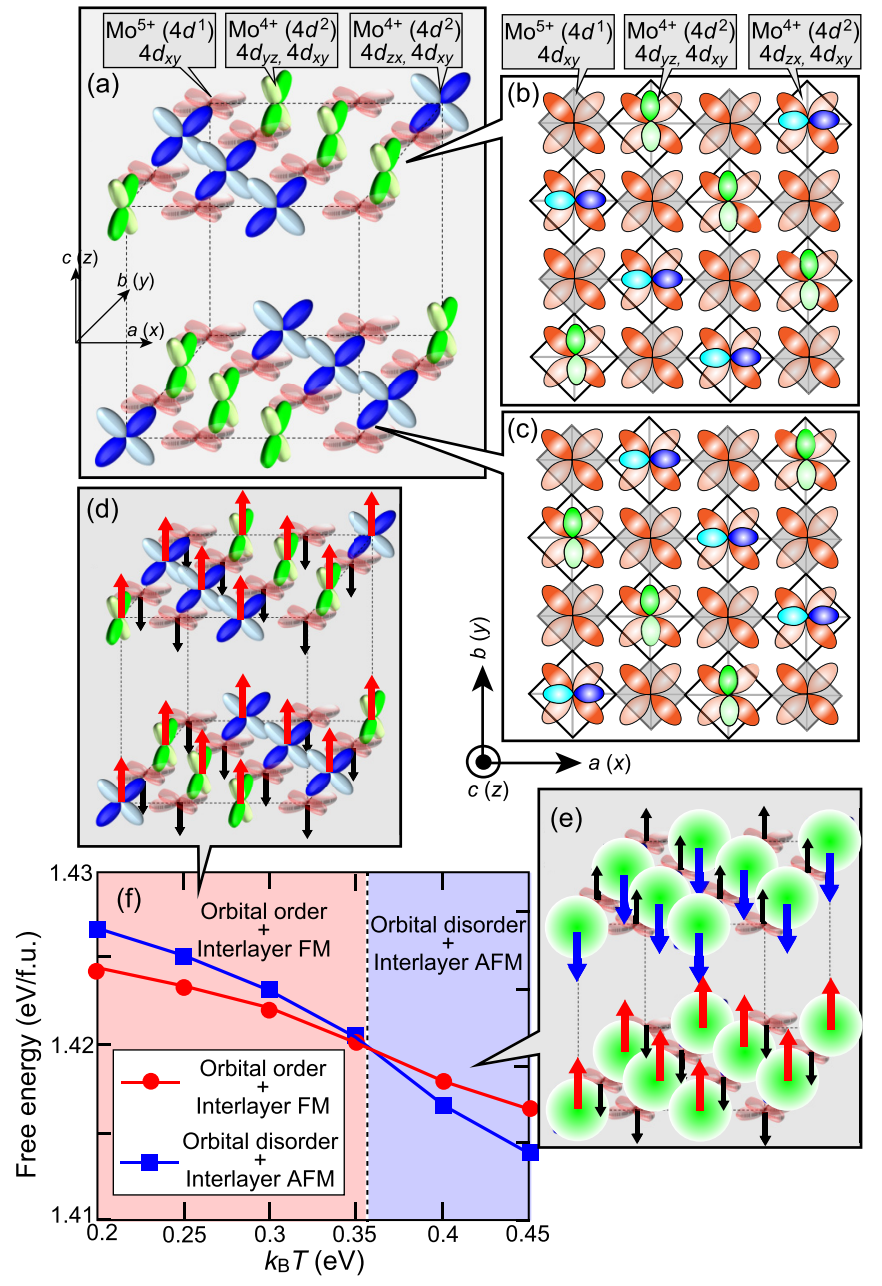

FIG. 17. (a) Theoretically predicted staggered orbital ordering in $\mathrm{La}_{5} \mathrm{Mo}_{4} \mathrm{O}_{16}$ at low $T$. The $4 d_{x y}$ orbital is occupied by an electron at every Mo site, whereas, at the $\mathrm{Mo}^{4+}\left(4 d^{2}\right)$ sites, one of the other two orbitals $\left(4 d_{y z}\right.$ or $\left.4 d_{z x}\right)$ is also occupied by an electron. Specifically, alternate occupations of $4 d_{y z}$ and $4 d_{z x}$ orbitals at the $\mathrm{Mo}^{4+}$ sites appear within the $a b$ plane, whereas alternate orbital stackings are realized along the $c$ axis. In this figure, the occupied $4 d_{x y}$ orbitals at the $\mathrm{Mo}^{4+}\left(4 d^{2}\right)$ sites are omitted for clarity. [(b) and (c)] Orbital configuration in each of two layers. (d), (e) Orbital and spin configurations in $\mathrm{La}_{5} \mathrm{Mo}_{4} \mathrm{O}_{16}$ when $H_{\text {ext }}=0$ : (d) the orbital ordered interlayer ferromagnetic state at low $T$ and (e) the orbital disordered interlayer antiferromagnetic state at intermediate $T$. (f) Free energies of these orbital-spin states as functions of $T k_{\mathrm{B}} T$, which show a phase transition from interlayer antiferromagnetic to interlayer ferromagnetic spin states as $T$ decreases. The change in the interlayer spin coupling is triggered by the orbital phase transition to the staggered orbital ordering.

favors the antiferromagnetic coupling in the $a b$ plane between adjacent $\mathrm{Mo}^{4+} S=1$ and $\mathrm{Mo}^{5+} S=1 / 2$ spins because of the uniform occupations of $4 d_{x y}$ orbitals at all Mo sites. On the other hand, the interlayer spin coupling is weakly ferromagnetic, which is in agreement with the experimental observation. This weak interlayer ferromagnetic coupling is caused by the second-order perturbation processes with respect to $t_{c}$ and the Hund's rule coupling in the staggered orbital configuration. As a result, the orbital ordered interlayer 


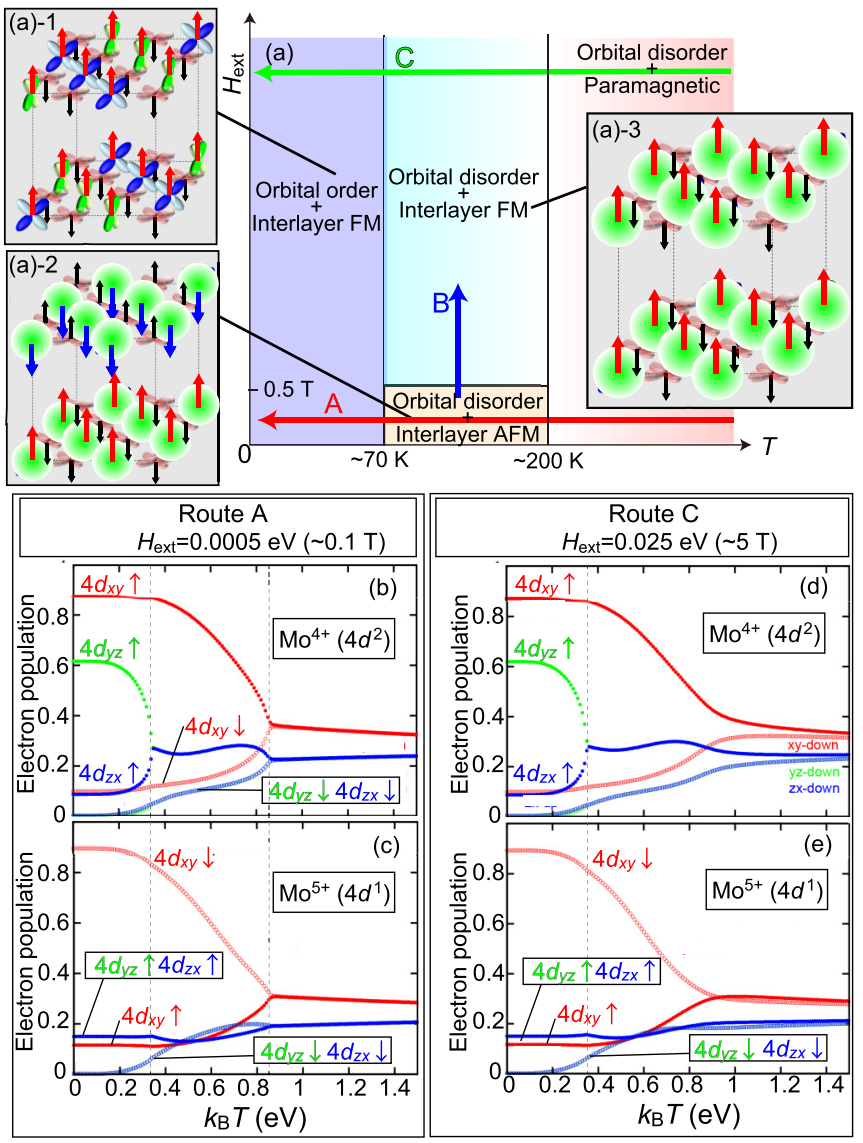

FIG. 18. (a) Schematic phase diagram of $\mathrm{La}_{5} \mathrm{Mo}_{4} \mathrm{O}_{16}$ in the plane of $T$ and $H_{\text {ext }}$. Possible routes of phase transitions are indicated by arrows (arrows A, B, and C). [(b) and (c)] Calculated temperature profiles of (b) electron populations of respective spin-orbital states at $\mathrm{Mo}^{4+}\left(4 d^{2}\right)$ site and (c) those at $\mathrm{Mo}^{5+}\left(4 d^{1}\right)$ site under a low magnetic field of $H_{\mathrm{ext}}=0.0005 \mathrm{eV}(\sim 0.1 \mathrm{~T})$. [(d) and (e)] Those under a high magnetic field of $H_{\mathrm{ext}}=0.025 \mathrm{eV}(\sim 5 \mathrm{~T})$. The profiles in (b) and (c) correspond to route A, which exhibit two successive phase transitions among three orbital-spin phases at low magnetic field: the orbital disordered paramagnetic phase at high $T$, the orbital disordered interlayer antiferromagnetic phase at intermediate $T$, and the orbital ordered interlayer ferromagnetic phase at low $T$. On the other hand, the profiles in (d) and (e) correspond to route $\mathrm{C}$, which exhibit only one phase transition at a high magnetic field from the high- $T$ orbital disordered phase to the low- $T$ orbital ordered phase, both in which the interlayer spin configuration is ferromagnetic.

ferromagnetic state [Fig. 17(d)] is realized at low $T$ when the magnetic field is absent or sufficiently low. In contrast, the orbitals are disordered at intermediate $T$, and both the intralayer and interlayer spin couplings are antiferromagnetic [Fig. 17(e)]. We observed a phase transition between these two phases upon the $T$ variation [Fig. 17(f)].

In Fig. 18(a), we show a schematic phase diagram of $\mathrm{La}_{5} \mathrm{Mo}_{4} \mathrm{O}_{16}$ in the plane of $T$ and $H_{\text {ext }}$. The aboveargued orbital disordered interlayer antiferromagnetic phase [Fig. 18(a)-2] is located in the region of low $H_{\text {ext }}$ and intermediate $T$. Starting from this phase, the system enters the orbital ordered interlayer ferromagnetic phase [Fig. 18(a)-1] as $T$ decreases along Arrow A. The energy difference between the interlayer antiferromagnetic state [Fig. 18(a)-2] and the interlayer ferromagnetic state [Fig. 18(a)-3] turned out to be small and hence, the interlayer spin configuration can easily be changed from antiferromagnetic to ferromagnetic by application of a weak magnetic field (along arrow B). This is consistent with the experimentally observed field-induced antiferromagnetic to ferromagnetic transition at $H_{\text {ext }} \sim 0.5 \mathrm{~T}$. Under a high $H_{\text {ext }}$ field, the system exhibits the orbital disorder-to-order phase transition as $T$ decreases along arrow $\mathrm{C}$, where the interlayer spin configuration remains ferromagnetic.

To clarify the nature of each phase transition, we calculate the temperature profiles of electron populations of each orbital-spin state at both $\mathrm{Mo}^{4+}$ and $\mathrm{Mo}^{5+}$ sites. Figures $18(\mathrm{~b})$ and $18(\mathrm{c})$ show those at $\mathrm{Mo}^{4+}$ and $\mathrm{Mo}^{5+}$ sites, respectively, at a very weak magnetic field of $H_{\text {ext }}=0.0005 \mathrm{eV}(\sim 0.5 \mathrm{~T})$. These profiles correspond to the $T$ variation along Arrow A in Fig. 18(a), where we observe three successive orbital-spin phases, i.e., the orbital disordered paramagnetic phase, the orbital disordered interlayer antiferromagnetic phase, and the orbital ordered interlayer ferromagnetic phase as $T$ decreases. With decreasing $T$, the electron populations of up-spin ( $\uparrow$ ) states and those of down-spin $(\downarrow)$ states become different for each Mo $t_{2 g}$ orbital upon the paramagnetic-to-ferrimagnetic phase transition. On the other hand, the electron populations of $4 d_{y z} \uparrow$ and $4 d_{z x} \uparrow$ orbitals are still equivalent even below this critical temperature, and their degeneracy is lifted at the lower critical temperature upon the emergence of orbital ordering. We observe similar profiles of electron populations also at a higher magnetic field of $H_{\text {ext }}=0.025 \mathrm{eV}(\sim 5 \mathrm{~T})$ in Figs. 18(d) and 18(e), which correspond to the $T$ variation along Arrow C in Fig. 18(a).

We next study the properties of magnetic domain walls in this compound. We first prepare an initial spin-orbital configuration with magnetic domain walls for iterative unrestricted Hartree-Fock calculations. We employ a system of $16 \times 8 \times 2$ sites with periodic boundary conditions, in which two different magnetic domains are involved, that is, one domain has $S=1 \mathrm{Mo}^{4+} \uparrow$ sites and $S=1 / 2 \mathrm{Mo}^{5+} \downarrow$ sites, whereas the other domain has $S=1 \mathrm{Mo}^{4+} \downarrow$ sites and $S=1 / 2 \mathrm{Mo}^{5+} \uparrow$ sites (see Fig. 19). Here, we consider the domain walls running along the $b(y)$ axis. To predict the orbital configuration inside the domain walls without any bias, we consider the orbital-disordered state with equal electron populations of three Mo $t_{2 g}$ orbitals. Starting with this initial state, we carried out iterations of the unrestricted Hartree-Fock calculations until all the order parameters are sufficiently converged. We also performed calculations starting from a uniform initial state without domain walls for comparison. In the present calculations, the interlayer transfer integral $t_{c}$ is set to be zero for simplicity, which is justified by the strong two-dimensional nature of $\mathrm{La}_{5} \mathrm{Mo}_{4} \mathrm{O}_{16}$. We confirmed that the results never change even quantitatively by this simplification.

Figures 20(a)-20(e) show calculated results associated with the density of states for a system without magnetic domains. Specifically, Fig. 20(a) shows the energy diagram in which low-lying 756 eigenenergies (among 1536 in total) obtained by diagonalizing the mean-field Hamiltonian are plotted in ascending order, whereas Fig. 20(b) shows the total density of states. These figures exhibit the appearance 


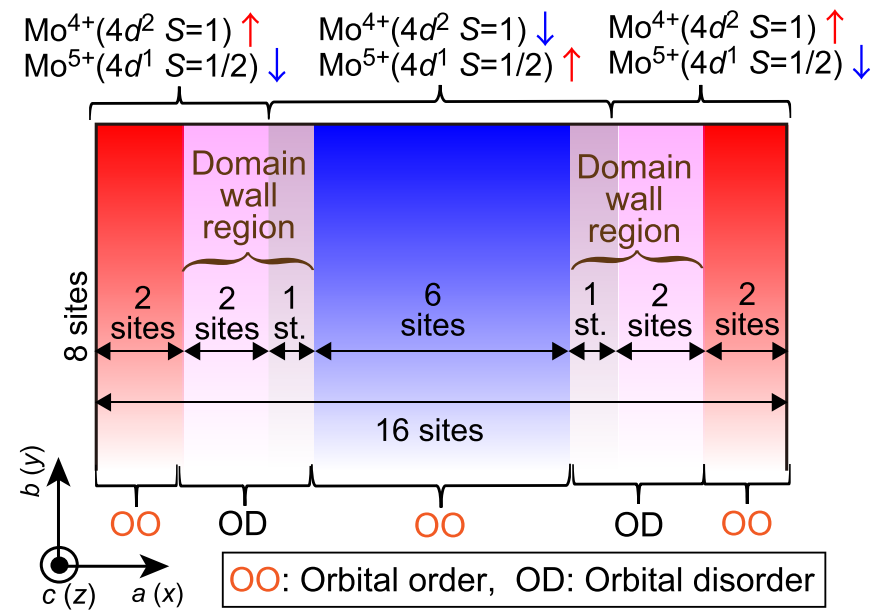

FIG. 19. Schematic illustration of initial spin-orbital configuration used for the iterative unrestricted Hartree-Fock calculation to study the properties of magnetic domain walls in $\mathrm{La}_{5} \mathrm{Mo}_{4} \mathrm{O}_{16}$ (see text).

of in-gap states around the Fermi level, with which we expect metallic conductivity. The partial densities of states in Figs. 20(c)-20(e) for the $4 d_{x y}, 4 d_{y z}$, and $4 d_{z x}$-orbital components indicate that these in-gap states have dominant $4 d_{y z}$ orbital components. On the other hand, Figs. 20(f)-20(g) show the results for a uniform system without domain walls, which exhibit no in-gap states, indicating that the system is insulating. From the comparisons between the two cases, we can conclude that the in-gap states and the metallic conductivity indeed originate from the magnetic domain walls.

In the presence of domain walls, we obtained eight in-gap states around the Fermi level for the present system size. Among them, four states are located slightly above the Fermi level and thus are nearly unoccupied, whereas the other four states are slightly below the Fermi level and thus are nearly occupied by electrons at low $T$. In Figs. 21(a), we show calculated spatial configurations of Mo $4 d$ spins $S_{i}$ in the system with domain walls. Here, $S_{i}$ on the $i$ th Mo site is given by

$$
S_{i}=\sum_{\ell, \mu, \sigma} \sigma\left(A_{i \mu \sigma}^{\ell}\right)^{*} A_{i \mu \sigma}^{\ell}
$$

where $A_{i \mu \sigma}^{\ell}$ is the eigenvector for the $l$ th eigenstate. We find that $S_{i}$ remains positive or negative along the $b(y)$ axis in the domain-wall region, indicating the emergence of onedimensional ferromagnetic spin chains inside the domain walls.

The emergence of metallic conductivity at the domain walls is attributable to these ferromagnetically aligned spins, which enhance the itinerant nature of the Mo $4 d$ electrons along the chains via the double exchange mechanism. This aspect can indeed be seen in the wavefunctions of the in-gap states around the Fermi level, which reflect the orbital-spin characters of the conductive electrons. Figures 21(b)-21(d) show the calculated three distribution probabilities $P_{\ell, i}^{\mathrm{c}}, P_{\ell, i}^{y z}$, and $P_{\ell, i}^{\sigma}$ for charge, $4 d_{y z}$ orbital, and spin at the $i$ th Mo site for the $\ell$ th eigenstate, which corresponds to one of the occupied
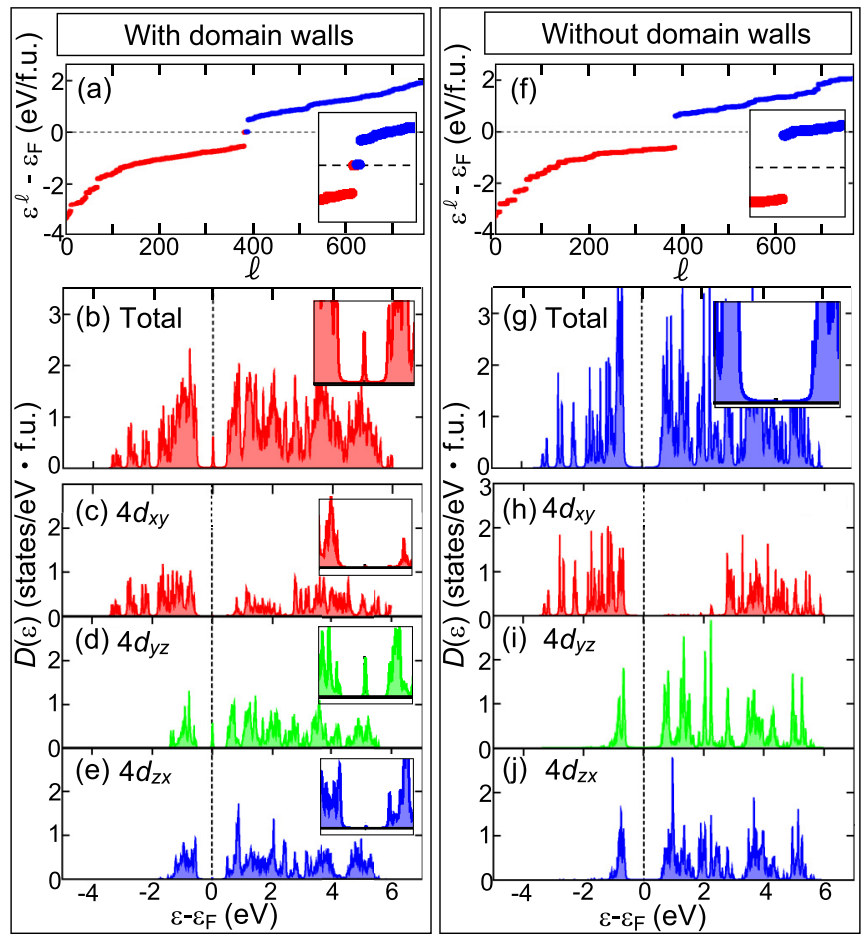

FIG. 20. [(a)-(e)] Calculated (a) energy diagram, (b) total density of states, and (c)-(e) partial densities of states for (c) $4 d_{x y}$, (d) $4 d_{y z}$, and (e) $4 d_{z x}$ orbital components for a system with domain walls. [(f) and (g)] Those for a system without domain walls. In the energy diagrams [(a) and (b)], the lower 756 eigenenergies $\varepsilon^{\ell}$ $(1 \leqslant \ell \leqslant 756)$ are arranged in ascending order. Insets in (a) and (b) are magnified views around the Fermi level of $350 \leqslant \ell \leqslant 450$ and $-1 \leqslant \varepsilon^{\ell}-\varepsilon_{\mathrm{F}}(\mathrm{eV}) \leqslant 1$, whereas insets in (b)-(e) and (g) are those of $-1 \leqslant \varepsilon^{\ell}-\varepsilon_{\mathrm{F}}(\mathrm{eV}) \leqslant 1$ and $0 \leqslant D(\varepsilon) \leqslant 1$. These figures clearly show the appearance of in-gap states with dominant $4 d_{y z}$ orbital component around the Fermi level when the system has domain walls.

in-gap states. They are respectively given by

$$
\begin{aligned}
P_{\ell, i}^{\mathrm{c}} & =\sum_{\mu, \sigma}\left(A_{i \mu, \sigma}^{\ell}\right)^{*} A_{i \mu, \sigma}^{\ell}, \\
P_{\ell, i}^{y z} & =\sum_{\sigma}\left(A_{i \mu=2, \sigma}^{\ell}\right)^{*} A_{i \mu=2, \sigma}^{\ell}, \\
P_{\ell, i}^{\sigma} & =\sum_{\mu, \sigma} \sigma\left(A_{i \mu, \sigma}^{\ell}\right)^{*} A_{i \mu, \sigma}^{\ell} .
\end{aligned}
$$

In fact, the eigenstates with these distribution patterns are fourfold degenerate, which are connected to each other by translation operation in the $b(y)$ direction. Figure 21(b) indicates that the wave function has a weight of the charge distribution probability $P_{\ell, i}^{\mathrm{c}}$ at the domain wall region, indicating that the in-gap states indeed originate from the Mo $4 d$ electrons on the magnetic domain walls. The distribution probabilities $P_{\ell, i}^{y z}$ and $P_{\ell, i}^{\sigma}$ indicate that the electronic structure in the domain walls possesses features favorable for metallic conductivity assisted by the double exchange mechanism. Firstly, the wavefunction of the in-gap state is composed of a dominant $4 d_{y z}$-orbital component [Fig. 21(b)], whereas the amounts of $4 d_{x y}$-orbital and $4 d_{z x}$-orbital components are 


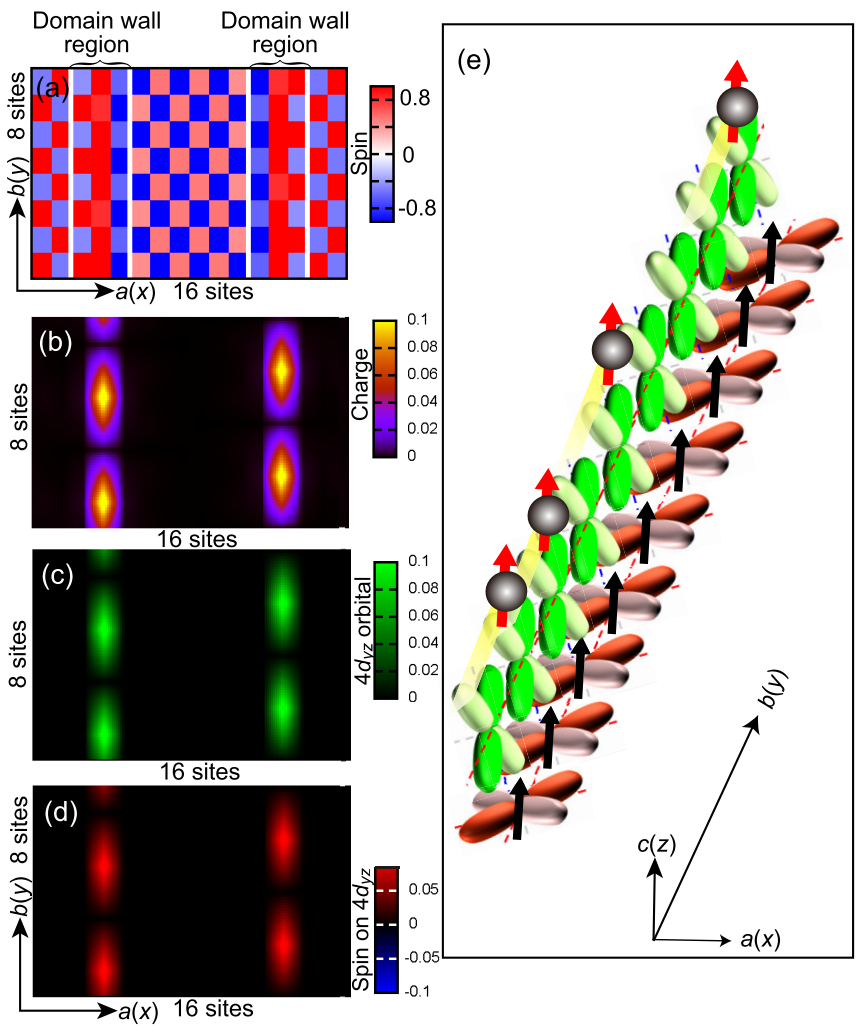

FIG. 21. (a) Calculated spatial spin configuration in the system with domain walls, inside of which ferromagnetic spin chains appear. [(b)-(d)] Calculated spatial distributions of (b) charge, (c) $4 d_{y z}$ orbital, and (d) $4 d_{y z}$-electron spin of an occupied in-gap state near the Fermi level, which appears in the system with domain walls. (e) Schematic illustration of the microscopic electronic structure of the conductive magnetic domain wall in $\mathrm{La}_{5} \mathrm{Mo}_{4} \mathrm{O}_{16}$. The electrons move along the one-dimensional array of Mo $4 d_{y z}$ orbitals along the $b(y)$ axis assisted by the double-exchange mechanism due to the ferromagnetically aligned Mo $4 d_{x y}$ spins.

negligibly small. Moreover, Fig. 21(c) as well as Fig. 21(b) indicates that coarsely and densely charged Mo sites are aligned along the domain walls, which enables the Mo $4 d$ electrons to move along the domain wall avoiding the influences of the Pauli exclusion principle. Secondly, the electron spins on the Mo $4 d_{y z}$-orbital chains are uniformly polarized in the direction parallel to the ferromagnetically aligned localized Mo $4 d_{x y}$ electron spins [Fig. 21(d)], with which the double-exchange mechanism assists the electron conduction, resulting in the metallic conductivity of the present multiorbital system in $\mathrm{La}_{5} \mathrm{Mo}_{4} \mathrm{O}_{16}$. The spin-orbital structures and the electron conductions in the domain walls of this material are schematically illustrated in Fig. 21(e).

\section{DISCUSSION}

On the basis of the present experimental and theoretical results, the magnetic/orbital states in $\mathrm{La}_{5} \mathrm{Mo}_{4} \mathrm{O}_{16}$ can be summarized as follows. Because of the strong antiferromagnetic interaction between the neighboring $\mathrm{Mo}^{4+}$ and $\mathrm{Mo}^{5+}$ spins within the $a b$ plane, together with the weak antiferromagnetic interaction between the $\mathrm{Mo}^{4+}$ moments on the neighboring layers via a nonmagnetic $\mathrm{Mo}_{2} \mathrm{O}_{10}$ pillar, the ferrimagnetic ordering within the plane and the antiferromagnetic ordering of the ferrimagnetic moments between the layers occur below $T_{N}=200 \mathrm{~K}$ [the IL-AF phase shown in Fig. 6(a)]. Because of the extremely small interlayer antiferromagnetic interaction, the antiferromagnetic alignment of the ferrimagnetic moments can be easily changed to the ferromagnetic alignment [the IL-F phase shown in Fig. 6(b)] when $H$ of $1 \mathrm{~T}$ is applied along the $c$ axis (Fig. 3). This metamagnetic phase transition causes a decrease in the resistivity along the $a, b$, and $c$ directions (negative magnetoresistance) with $|\Delta \rho / \rho|$ of several percent, as shown in Fig. 5. Such a metamagnetic phase transition does not occur when $H$ is applied along the $a b$ plane (Fig. 3) because of the strong Ising anisotropy of the Mo spins along the $c$ axis.

On the other hand, below $T_{s 2}=95 \mathrm{~K}$ and $T_{s 1}=60 \mathrm{~K}$, orbital ordering occurs for the Mo ions. In the Hartree-Fock calculation, this orbital ordering occurs only for the $\mathrm{Mo}^{4+}$ ions in the $4 d^{2}$ electron configuration in such a way that the first electron occupies the $x y$ orbital whereas the second electron occupies the $y z$ and $z x$ orbitals alternately for the neighboring $\mathrm{Mo}^{4+}$ ions both within the $a b$ plane and also along the $c$ axis, as shown in Figs. 17(a)-17(c). Such antiferro-orbital ordering causes the ferromagnetic interaction along the $c$ axis, and the magnetic state changes from the antiferromagnetic alignment to the ferromagnetic alignment of the ferrimagnetic moments along the $c$ axis (the D-IL-F phase).

This change in the magnetic structure with $T$ leads to a domain structure with the upward and downward magnetizations [Fig. 6(c)]. Here, the boundary between a domain with upward magnetization, where $d^{2}$ is upward and $d^{1}$ is downward, and that with downward magnetization, where $d^{2}$ is downward and $d^{1}$ is upward, should have a ferromagnetic alignment of the $d^{2}$ and $d^{1}$ moments across the domain boundary, as shown in Fig. 22(a). According to the calculation, however, this is not the spin configuration at the boundary of the real magnetic domains, but the spin configuration shown in Fig. 22(b), where the Mo spins are ferromagnetically aligned within the domain wall along the wall direction, is realized. This spin configuration is favored by the double-exchange mechanism of ferromagnetism with a metallic conduction along the domain wall, which is caused by the change in the occupation of the $d$ electrons in each orbital (less $d$ electrons in the $x y$ state but more in the $y z$ state along the domain wall). Other than such ferromagnetic configuration of the Mo spins along the domain wall, the number of the wrong bonds, i.e., the ferromagnetic configuration of the neighboring spins despite the antiferromagnetic interaction represented by the arrows in Fig. 22(b), is the same as that in (a). With applied $H$, these magnetic domains and the domain-wall conduction disappear, resulting in the nonvolatile positive magnetoresistance, as shown in Fig. 9.

Experimentally, there are two phase transitions at $T_{s 2}=$ $95 \mathrm{~K}$ and $T_{s 1}=60 \mathrm{~K}$, although only one phase transition for the orbital ordering is predicted theoretically. According to the result of $M(T)$ with $H=5 \mathrm{~T}$ along the $c$ axis shown in Fig. 2(d), where the ferrimagnetic moment at each layer is polarized in the same direction, $M$ increases below $T_{s 2}$ but decreases below $T_{s 1}$. This means that the size of the ferrimagnetic moment at each layer also increases below $T_{s 2}$ but decreases below $T_{s 1}$. Assuming that the size of any magnetic 
(a)

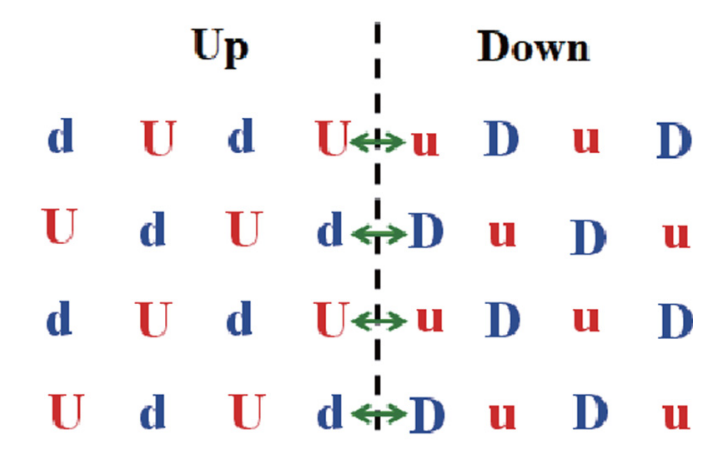

(b)

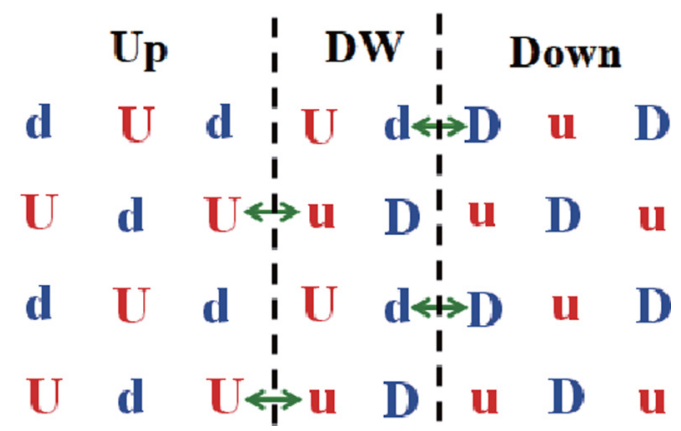

FIG. 22. Schematics of (a) the simple domain wall and (b) domain wall obtained by the Hartree-Fock calculation. Capital "U" and "D" correspond to the up and down $d^{2}$ spins, respectively, whereas small "u" and "d" correspond to the up and down $d^{1}$ spins, respectively. Arrows show the "wrong" spin configuration in terms of the antiferromagnetic interaction.

moments should increase with decreasing $T$, it is reasonable to consider that the longer moment of the $\mathrm{Mo}^{4+}$ becomes further longer below $T_{s 2}$ and the shorter moment of the $\mathrm{Mo}^{5+}$ becomes longer below $T_{s 1}$, resulting in such $T$ dependence of $M$ as observed experimentally. The latter is not reproduced in the Hartree-Fock calculation probably because the effect of fluctuation is not sufficiently taken into account in the theory.

The orbital ordering of the Mo ions induces the anomalies in the strain at $T_{s 2}$ and $T_{s 1}$ along the $b$ and $c$ axes but not along the $a$ axis, as shown in Fig. 11(a). The orbital ordering of the Mo $4 d$ states induces the distortion of the $\mathrm{MoO}_{6}$ octahedra, and this leads to the change in the $b$ lattice constant along which the Mo-O-Mo angle is $180^{\circ}$. On the other hand, the $a$ lattice constant barely changes probably because the contribution to the change in the $a$ lattice constant by the distortion of the $\mathrm{MoO}_{6}$ octahedra is canceled out by the change in the Mo-O-Mo angle $\left(<180^{\circ}\right.$, as shown in Fig. 1). We speculate that the change in the $c$ lattice constant is a secondary effect of the change in the $b$ lattice constant, with a tendency to keep the lattice volume unchanged with the structural anomalies at $T_{s 2}$ and $T_{s 1}$.

It should be pointed out that the size of the magnetic domains in the D-IL-F phase is relatively small despite the large Ising-type anisotropy of the Mo moments in $\mathrm{La}_{5} \mathrm{Mo}_{4} \mathrm{O}_{16}$. For example, in the case of $R_{2} \mathrm{Ir}_{2} \mathrm{O}_{7}$, a typical size of the magnetic domains is several micrometers [8], which is two orders of magnitude larger in length than that of the present compound $(\sim 100 \mathrm{~nm})$. Such large magnetic domains in $R_{2} \mathrm{Ir}_{2} \mathrm{O}_{7}$ are consistent with the large Ising-type anisotropy of the Ir moments, which leads to a large energy cost at the domain wall (proportional to the square root of the magnetic anisotropy energy), resulting in a larger size of the magnetic domains (smaller ratio of the area of the domain boundaries to the volume of the domain). On the other hand, smaller domains in $\mathrm{La}_{5} \mathrm{Mo}_{4} \mathrm{O}_{16}$ despite a large Ising anisotropy are caused by the fact that the magnetic phase transition induced by the orbital ordering occurs at a much lower than $T_{\mathrm{N}}$. Thus, the growth rate of the ferrimagnetic nuclei, which is dominated by the activation energy corresponding to the energy barrier to flip the Mo moments with Ising anisotropy, is extremely small. In other words, the domain structures of the D-IL-F phase below $T_{s 1}$ in this compound are not formed as an equilibrium state but in an inequilibrium manner. Such an inequilibrium character results in the disordered structure of the magnetic domains, and it manifests itself in the anomalous relaxation behavior of the resistivity and magnetization [21] and also in the memory effect of the magnetization below $T_{s 1}$ (Fig. 15). On the other hand, the magnetic domains in the IL-AF phase are formed in the equilibrium state and thus, their size will be much larger than that in the D-IL-F phase, resulting in the negligible domain-wall conduction in the IL-AF phase.

\section{SUMMARY}

We studied $\mathrm{La}_{5} \mathrm{Mo}_{4} \mathrm{O}_{15}$, where the $\mathrm{Mo}^{4+}$ ions $\left(4 d^{2}\right)$ and $\mathrm{Mo}^{5+}$ ions $\left(4 d^{1}\right)$ are arranged in a checkerboard manner on a quasisquare lattice, both experimentally and theoretically. We found that the $4 d^{2}$ and $4 d^{1}$ spins are ferrimagnetically ordered within a plane of the square lattice below $T_{\mathrm{N}}=200 \mathrm{~K}$, and the ferrimagnetic moments are aligned antiferromagnetically between the layers above $T_{s 1}=60 \mathrm{~K}$, whereas they are changed to the ferromagnetic alignment below $T_{s 1}$. By theoretical calculation, we found that the change in the magnetic structure below $T_{s 1}$ is caused by the orbital ordering of the Mo ions. The existence of the two anomalies at $T_{s 2}=95 \mathrm{~K}$ and $T_{s 1}=60 \mathrm{~K}$ experimentally observed is likely caused by the orbital ordering of not only $\mathrm{Mo}^{4+}\left(d^{2}\right)$ but also $\mathrm{Mo}^{5+}\left(d^{1}\right)$. It was also found that there are magnetic domains with spontaneous magnetization in the length scale of $\sim 100 \mathrm{~nm}$ below $T_{s 1}$, which is responsible for the memory effect in the magnetization. The nonvolatile positive magnetoresistance observed below $T_{s 1}$ is caused by the disappearance of the electrical conduction along the boundaries of such magnetic domains (domain-wall conduction) when $H \sim 1 \mathrm{~T}$ is applied along the $c$ axis. We found by theoretical calculation that in-gap states appear around the Fermi level associated with the formation of the domain walls, causing the domain-wall conduction.

\section{ACKNOWLEDGMENTS}

We thank R. Kajimoto, H. Wadati, M. Nakamura, and T. Mizokawa for enlightening discussions. T.K. was supported by JSPS KAKENHI Grant No. 19H01853 and Waseda University Grant for Special Research Projects (Project No. 2018B-184). M.M. was supported by JSPS KAKENHI (Grants No. 17H02924, No. 16H06345, No. 19H00864, No. 
19K21858, and No. 20H00337) and Waseda University Grant for Special Research Projects (Projects No. 2019C-253 and No. 2020C-269). Y.S. and M.I. were supported by JSPS KAKENHI (Grants No. 19H01837 and No. 19H05824). F.K. was supported by JSPS KAKENHI (Grants No. 18H01168 and No. 18H05225).

\section{APPENDIX A: DETAILS OF THE HARTREE-FOCK CALCULATION}

We apply the unrestricted Hartree-Fock approximation to these Coulomb-interaction terms to obtain the following mean-field Hamiltonian:

$$
\begin{aligned}
& \mathcal{H}=\sum_{i, j} \sum_{\mu, \nu} \sum_{\sigma} t_{i, \mu ; j, \nu} c_{i \mu \sigma}^{\dagger} c_{j \nu \sigma}+\sum_{i} \sum_{\mu, \nu} \sum_{\sigma, \sigma^{\prime}} \lambda_{\mu \sigma ; \nu \sigma^{\prime}} c_{i \mu \sigma}^{\dagger} c_{i v \sigma^{\prime}}+\sum_{i \in \mathrm{Mo}^{++}} \sum_{\mu} \Delta_{\mathrm{st}} c_{i \mu \sigma}^{\dagger} c_{i \mu \sigma}+\sum_{i} \sum_{\mu \in x y} \Delta_{x y} c_{i \mu \sigma}^{\dagger} c_{i \mu \sigma} \\
& \left.+H_{\mathrm{ext}} \sum_{i} \sum_{\mu}\left(\left\langle c_{i \mu \uparrow}^{\dagger} c_{i \mu \uparrow}\right\rangle-\left\langle c_{i \mu \downarrow}^{\dagger} c_{i \mu \downarrow}\right)\right\rangle\right)+u \sum_{i} \sum_{\mu}\left(\left\langle c_{i \mu \uparrow}^{\dagger} c_{i \mu \uparrow}\right\rangle c_{i \mu \downarrow}^{\dagger} c_{i \mu \downarrow}+c_{i \mu \uparrow}^{\dagger} c_{i \mu \uparrow}\left\langle c_{i \mu \downarrow}^{\dagger} c_{i \mu \downarrow}\right\rangle\right) \\
& +u^{\prime} \sum_{i} \sum_{\mu \neq \nu}\left(\left\langle c_{i \mu \uparrow}^{\dagger} c_{i \mu \uparrow}\right\rangle c_{i \nu \downarrow}^{\dagger} c_{i \nu \downarrow}+c_{i \mu \uparrow}^{\dagger} c_{i \mu \uparrow}\left\langle c_{i \nu \downarrow}^{\dagger} c_{i \nu \downarrow}\right\rangle\right)+\left(u^{\prime}-j\right) \sum_{i} \sum_{\mu>\nu} \sum_{\sigma}\left(\left\langle c_{i \mu \sigma}^{\dagger} c_{i \mu \sigma}\right\rangle c_{i \nu \sigma}^{\dagger} c_{i \nu \sigma}+c_{i \mu \sigma}^{\dagger} c_{i \mu \sigma}\left\langle c_{i \nu \sigma}^{\dagger} c_{i \nu \sigma}\right\rangle\right) \\
& -\left(u^{\prime}-j\right) \sum_{i} \sum_{\mu>\nu} \sum_{\sigma}\left(\left\langle c_{i \mu \sigma}^{\dagger} c_{i v \sigma}\right\rangle c_{i \nu \sigma}^{\dagger} c_{i \mu \sigma}+c_{i \mu \sigma}^{\dagger} c_{i \nu \sigma}\left\langle c_{i \nu \sigma}^{\dagger} c_{i \mu \sigma}\right\rangle\right)+j^{\prime} \sum_{i} \sum_{\mu \neq \nu}\left(\left\langle c_{i \mu \uparrow}^{\dagger} c_{i v \uparrow}\right\rangle c_{i \nu \downarrow}^{\dagger} c_{i \mu \downarrow}+c_{i \mu \uparrow}^{\dagger} c_{i \nu \uparrow}\left\langle c_{i \nu \downarrow}^{\dagger} c_{i \mu \downarrow}\right\rangle\right) \\
& +j \sum_{i} \sum_{\mu \neq \nu}\left(\left\langle c_{i \mu \uparrow}^{\dagger} c_{i \nu \uparrow}\right\rangle c_{i \mu \downarrow}^{\dagger} c_{i \nu \downarrow}+c_{i \mu \uparrow}^{\dagger} c_{i \nu \uparrow}\left\langle c_{i \mu \downarrow}^{\dagger} c_{i \nu \downarrow}\right\rangle\right)-U_{0},
\end{aligned}
$$

where

$$
\begin{aligned}
U_{0}= & u \sum_{i} \sum_{\mu}\left\langle c_{i \mu \uparrow}^{\dagger} c_{i \mu \uparrow}\right\rangle\left\langle c_{i \mu \downarrow}^{\dagger} c_{i \mu \downarrow}\right\rangle+u^{\prime} \sum_{i} \sum_{\mu \neq \nu}\left\langle c_{i \mu \uparrow}^{\dagger} c_{i \mu \uparrow}\right\rangle\left\langle c_{i \nu \downarrow}^{\dagger} c_{i \nu \downarrow}\right\rangle+\left(u^{\prime}-j\right) \sum_{i} \sum_{\mu>\nu} \sum_{\sigma}\left\langle c_{i \mu \sigma}^{\dagger} c_{i \mu \sigma}\right\rangle\left\langle c_{i \nu \sigma}^{\dagger} c_{i \nu \sigma}\right\rangle \\
& -\left(u^{\prime}-j\right) \sum_{i} \sum_{\mu>\nu} \sum_{\sigma}\left\langle c_{i \mu \sigma}^{\dagger} c_{i \nu \sigma}\right\rangle\left\langle c_{i \nu \sigma}^{\dagger} c_{i \mu \sigma}\right\rangle+j^{\prime} \sum_{i} \sum_{\mu \neq \nu}\left\langle c_{i \mu \uparrow}^{\dagger} c_{i \nu \uparrow}\right\rangle\left\langle c_{i \nu \downarrow}^{\dagger} c_{i \mu \downarrow}\right\rangle+j \sum_{i} \sum_{\mu \neq \nu}\left\langle c_{i \mu \uparrow}^{\dagger} c_{i \nu \uparrow}\right\rangle\left\langle c_{i \mu \downarrow}^{\dagger} c_{i \nu \downarrow}\right\rangle .
\end{aligned}
$$

For the numerical calculations, we first input initial expectation values to the mean fields $\left\langle c_{i \mu \sigma}^{\dagger} c_{i v \sigma^{\prime}}\right\rangle$ in the Coulomb-interaction terms by assuming a certain spin and orbital configuration. Then, we diagonalize the matrix of the mean-field Hamiltonian and obtain eigenenergies $\varepsilon^{\ell}$ and corresponding eigenvectors $A_{i \mu \sigma}^{\ell}$. Then, we determine the chemical potential $\mu$ so as to satisfy the relation

$$
N_{\text {ele. }}=\sum_{\ell=1}^{N} \sum_{i, \mu, \sigma} \frac{1}{1+e^{\beta\left(\varepsilon^{\ell}-\mu\right)}},
$$

where $\beta=1 / k_{\mathrm{B}} T$. Here, $N_{\text {ele. }}$ and $N$ are the total number of electrons and the dimension of eigenvectors, respectively.

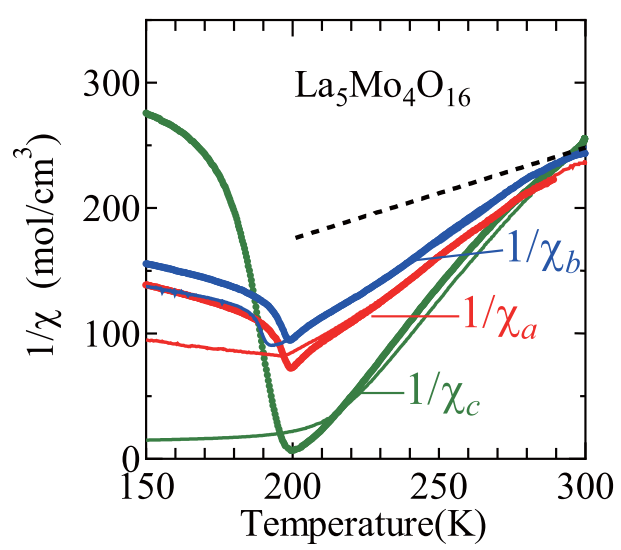

FIG. 23. Inverse magnetic susceptibility for $\mathrm{La}_{5} \mathrm{Mo}_{4} \mathrm{O}_{16}$ with $H=0.1 \mathrm{~T}$ (dots) and $5 \mathrm{~T}$ (thin solid lines) along the $a$ (red), $b$ (blue), and $c$ (green) axis.
Using the obtained eigenvectors and chemical potential, we calculate new expectation values of the mean fields:

$$
\left\langle c_{i \mu \sigma}^{\dagger} c_{i v \sigma^{\prime}}\right\rangle=\sum_{\ell=1}^{N} \frac{\left(A_{i \mu \sigma}^{\ell}\right)^{*} A_{i v \sigma^{\prime}}^{\ell}}{1+e^{\beta\left(\varepsilon^{\ell}-\mu\right)}} .
$$

We again input these newly calculated expectation values to the mean-field Hamiltonian and repeat the above procedures. We continue this iterative self-consistency calculations until all the order parameters $\left\langle c_{i \mu \sigma}^{\dagger} c_{i \nu \sigma^{\prime}}\right\rangle$ are sufficiently converged. After the convergence, we calculate the total energy $E$ and the free energy $F$ for the eigenstate:

$$
\begin{gathered}
E=\sum_{\ell=1}^{N} \frac{\varepsilon^{\ell}}{1+e^{\beta\left(\varepsilon^{\ell}-\mu\right)},} \\
F=-\frac{1}{\beta} \sum_{\ell=1}^{N} \log \left(1+e^{\beta\left(\varepsilon^{\ell}-\mu\right)}\right)+\mu n .
\end{gathered}
$$

This formalism can be used even for the ground-state calculations at $T=0$ by the following simple replacement:

$$
\frac{1}{1+e^{\beta\left(\varepsilon^{\ell}-\mu\right)}} \rightarrow 1-\theta\left(\varepsilon^{\ell}-\varepsilon_{\mathrm{F}}\right),
$$

where $\varepsilon_{\mathrm{F}}$ is the Fermi energy. The model parameters for the molybdenum transition-metal oxides were evaluated in several theoretical studies based on first-principles calculations and model calculations [36,37]. 


\section{APPENDIX B: MAGNETIZATION ABOVE $\boldsymbol{T}_{N}$}

Figure 23 shows the inverse magnetic susceptibility for $\mathrm{La}_{5} \mathrm{Mo}_{4} \mathrm{O}_{16}$ at $H=0.1 \mathrm{~T}$ (dots) and $5 \mathrm{~T}$ (thin solid lines) along the $a$ ( $\chi_{a}$, red $), b\left(\chi_{b}\right.$, blue), and $c$ axis $\left(\chi_{c}\right.$, green). The dashed line represents the slope of the inverse magnetic susceptibility corresponding to a $S=1$ and a $S=$ $1 / 2$ spin in a unit cell $\left(C=1.38 \mathrm{~K} \mathrm{~cm}^{3} / \mathrm{mol}\right)$. As can be seen, the behavior at $H=0.1 \mathrm{~T}$ and that at $5 \mathrm{~T}$ are almost the same for each of $\chi_{a}, \chi_{b}$, and $\chi_{c}$, but they are slightly different between the three. Furthermore, they are discernibly different from the theoretical slope of the inverse magnetic susceptibility with $S=1$ and $S=1 / 2$ shown by the dashed line in Fig. 23 below $\sim 280 \mathrm{~K}$. Note that in ferrimagnets, the inverse magnetic susceptibility behaves in such a way that it obeys a Curie-Weiss law $\chi=C /(T+\theta)$ with $\theta>0$ at high temperatures $(T>$ $\left.T^{*}\right)$, but for $T_{c}<T<T^{*}, \chi$ is deviated to larger values $(1 / \chi$ is deviated to smaller values) from the Curie-Weiss law even within the mean-field approximation [3]. The temperature dependence of the magnetic susceptibility for the present compound is consistent with that of ferrimagnets with $T^{*} \sim 280 \mathrm{~K}$.
[1] G. Catalan, J. Seidel, R. Ramesh, and J. F. Scott, Domain wall nanoelectronics, Rev. Mod. Phys. 84, 119 (2012).

[2] J. F. Scott, Ferroelectric Memories (Springer Nature, Berlin, 2000).

[3] S. Chikazumi, Physics of Ferromagnetism (Oxford University Press, London, 2009).

[4] J. Seidel, L. W. Martin, Q. He, Q. Zhan, Y.-H. Chu, A. Rother, M. E. Hawkridge, P. Maksymovych, P. Yu, M. Gajek, N. Balke, S. V. Kalinin, S. Gemming, F. Wang, G. Catalan, J. F. Scott, N. A. Spaldin, J. Orenstein, and R. Ramesh, Conduction at domain walls in oxide multiferroics, Nat. Mater. 8, 229 (2009).

[5] J. Seidel, P. Maksymovych, Y. Batra, A. Katan, S.-Y. Yang, Q. He, A. P. Baddorf, S. V. Kalinin, C.-H. Yang, J.-C. Yang, Y.-H. Chu, E. K. H. Salje, H. Wormeester, M. Salmeron, and R. Ramesh, Domain Wall Conductivity in La-Doped $\mathrm{BiFeO}_{3}$, Phys. Rev. Lett. 105, 197603 (2010).

[6] D. Meier, J. Seidel, A. Cano, K. Delaney, Y. Kumagai, M. Mostovoy, N. A. Spaldin, R. Ramesh, and M. Fiebig, Anisotropic conductance at improper ferroelectric domain walls, Nat. Mater. 11, 284 (2012).

[7] W. Wu, Y. Horibe, N. Lee, S.-W. Cheong, and J. R. Guest, Conduction of Topologically Protected Charged Ferroelectric Domain Walls, Phys. Rev. Lett. 108, 077203 (2012).

[8] E. Y. Ma, Y. T. Cui, K. Ueda, S. Tang, K. Chen, N. Tamura, P. M. Wu, J. Fujioka, Y. Tokura, and Z. X. Shen, Mobile metallic domain walls in an all-in-all-out magnetic insulator, Science 350, 538 (2015).

[9] K. Ueda, J. Fujioka, Y. Takahashi, T. Suzuki, S. Ishiwata, Y. Taguchi, M. Kawasaki, and Y. Tokura, Anomalous domain-wall conductance in pyrochlore-type $\mathrm{Nd}_{2} \mathrm{Ir}_{2} \mathrm{O}_{7}$ on the verge of the metal-insulator transition, Phys. Rev. B 89, 075127 (2014).

[10] Y. Yamaji and M. Imada, Metallic Interface Emerging at Magnetic Domain Wall of Antiferromagnetic Insulator: Fate of Extinct Weyl Electrons, Phys. Rev. X 4, 021035 (2014).

[11] H. T. Hirose, J. Yamaura, and Z. Hiroi, Robust ferromagnetism carried by antiferromagnetic domain walls, Sci. Rep. 7, 42440 (2017).

[12] K. Yasuda, M. Mogi, R. Yoshimi, A. Tsukazaki, K. S. Takahashi, M. Kawasaki, F. Kagawa, and Y. Tokura, Quantized chiral edge conduction on domain walls of a magnetic topological insulator, Science 358, 1311 (2017).

[13] W. H. McCarroll, C. Darling, and G. Jakubicki, Synthesis of reduced complex oxides of molybdenum by fused salt electrolysis, J. Solid State Chem. 48, 189 (1983).
[14] M. Ledesert, P. Labbe, W. H. McCarroll, H. Leligny, and B. Raveau, $\mathrm{La}_{5} \mathrm{Mo}_{4} \mathrm{O}_{16}$ : A new structural type related to perovskite with extremely short mo-mo bonds, J. Solid State Chem. 105, 143 (1993).

[15] K. Ramanujachary, M. Greenblatt, W. H. McCarroll, and J. B. Goodenough, Anomalous electrical and magnetic properties of a new quasi-low-dimensional mixed-valent molybdenum-cluster compound, Mat. Res. Bull. 28, 1257 (1993).

[16] K. Ramanujachary, S. E. Lofland, W. H. McCarroll, T. J. Emge, M. Greenblatt, and M. Croft, Substitutional effects of 3d transition metals on the magnetic and structural properties of quasi-two-dimensional $\mathrm{La}_{5} \mathrm{Mo}_{4} \mathrm{O}_{16}$, J. Solid State Chem. 164, 60 (2002).

[17] S. E. Lofland, T. Scabarozi, K. V. Ramanujachary, and W. H. McCarroll, Unusual magnetic properties of $\mathrm{La}_{5} \mathrm{Mo}_{4} \mathrm{O}_{16}, \mathrm{~J}$. Magn. Magn. Mater. 260, 184 (2003).

[18] F. Ramezanipour, S. Derakhshan, J. E. Greedan, and L. M. D. Cranswick, Synthesis, crystal structure and magnetic properties of a new pillared perovskite $\mathrm{La}_{5} \mathrm{Mo}_{2.75} \mathrm{~V}_{1.25} \mathrm{O}_{16}$, J. Solid State Chem. 181, 3366 (2008).

[19] K. Kobayashi and T. Katsufuji, High-temperature interlayer magnetoresistance in $\mathrm{La}_{5} \mathrm{Mo}_{4} \mathrm{O}_{16}$, Phys. Rev. B 83, 100411(R) (2011).

[20] T. Konishi, K. Kobayashi, and T. Katsufuji, Long-time relaxation of the magnetization in a pure crystal magnet $\mathrm{La}_{5} \mathrm{Mo}_{4} \mathrm{O}_{16}$, Phys. Rev. B 92, 020419(R) (2015).

[21] Y. Mizuno, T. Hasegawa, and T. Katsufuji, Anomalous relaxation behavior in the resistivity and magnetization of $\mathrm{La}_{5} \mathrm{Mo}_{4} \mathrm{O}_{16}$, Phys. Rev. B 95, 224436 (2017).

[22] K. Iida, R. Kajimoto, Y. Mizuno, K. Kamazawa, Y. Inamura, A. Hoshikawa, Y. Yoshida, T. Matsukawa, T. Ishigaki, Y. Kawamura, S. Ibuka, T. Yokoo, S. Itoh, and T. Katsufuji, Timeof-flight elastic and inelastic neutron scattering studies on the localized $4 \mathrm{~d}$ electron layered perovskite $\mathrm{La}_{5} \mathrm{Mo}_{4} \mathrm{O}_{16}$, J. Phys. Soc. Jpn. 86, 064803 (2017).

[23] C. Wiebe, A. Gourrier, T. Langet, J. Britten, and J. Greedan, Synthesis, structure, and magnetic behavior of $\mathrm{La}_{5} \mathrm{Re}_{3} \mathrm{MnO}_{16}$ : A new perovskite-like material, J. Solid State Chem. 151, 31 (2000).

[24] L. Chi, A. Green, R. Hammond, C. Wiebe, and J. Greedan, Synthesis, structure and magnetic properties of the pillared perovskites $\mathrm{La}_{5} \mathrm{Re}_{3} \mathrm{MO}_{16}(\mathrm{M}=\mathrm{Mg}, \mathrm{Fe}, \mathrm{Co}, \mathrm{Ni})$, J. Solid State Chem. 170, 165 (2003). 
[25] L. Chi, I. Swainson, and J. Greedan, Synthesis, crystal structure and magnetic properties of an Os-containing pillared perovskite $\mathrm{La}_{5} \mathrm{Os}_{3} \mathrm{MnO}_{16}$, J. Solid State Chem. 177, 3086 (2004).

[26] H. L. Cuthbert, J. E. Greedan, and L. Cranswick, Investigations of the magnetic properties and structures of the pillared perovskites, $\mathrm{La}_{5} \mathrm{Re}_{3} \mathrm{MO}_{16}(\mathrm{M}=\mathrm{Co}, \mathrm{Ni})$, J. Solid State Chem. 179, 1938 (2006).

[27] K. Momma and F. Izumi, Vesta 3 for three-dimensional visualization of crystal, volumetric and morphology data, Appl. Crystallogr. 44, 1272 (2011).

[28] M. Nakamura, D. Morikawa, X. Yu, F. Kagawa, T. Arima, Y. Tokura, and M. Kawasaki, Emergence of topological hall effect in half-metallic manganite thin films by tuning perpendicular magnetic anisotropy, J. Phys. Soc. Jpn. 87, 074704 (2018).

[29] K. Jonason, E. Vincent, J. Hammann, J. P. Bouchaud, and P. Nordblad, Memory and Chaos Effects in Spin Glasses, Phys. Rev. Lett. 81, 3243 (1998).

[30] S. Bedanta and W. Kleemann, Supermagnetism, J. Phys. D: Applied Physics 42, 013001 (2008).
[31] Y. Sun, M. B. Salamon, K. Garnier, and R. S. Averback, Memory Effects in an Interacting Magnetic Nanoparticle System, Phys. Rev. Lett. 91, 167206 (2003).

[32] M. Sasaki, P. E. Jönsson, H. Takayama, and P. Nordblad, Comment on "memory Effects in an Interacting Magnetic Nanoparticle System", Phys. Rev. Lett. 93, 139701 (2004).

[33] R. K. Zheng, H. Gu, and X. X. Zhang, Comment on "Memory Effects in an Interacting Magnetic Nanoparticle System", Phys. Rev. Lett. 93, 139702 (2004).

[34] T. Mizokawa and A. Fujimori, Electronic structure and orbital ordering in perovskite-type $3 \mathrm{~d}$ transition-metal oxides studied by hartree-fock band-structure calculations, Phys. Rev. B 54, 5368 (1996).

[35] J. Kanamori, Electron correlation and fer romagnetism of transition metals., Prog. Theor. Phys. 30, 275 (1963).

[36] I. V. Solovyev, Effects of crystal structure and on-site coulomb interactions on the electronic and magnetic structure of $A_{2} \mathrm{Mo}_{2} \mathrm{O}_{7}(a=\mathrm{Y}, \mathrm{Gd}$, and Nd) pyrochlores, Phys. Rev. B 67, 174406 (2003).

[37] H. Shinaoka, Y. Motome, T. Miyake, and S. Ishibashi, Spinorbital frustration in molybdenum pyrochlores $A_{2} \mathrm{Mo}_{2} \mathrm{O}_{7}(A=$ rare earth), Phys. Rev. B 88, 174422 (2013). 